\title{
Isotropic-nematic phase equilibria of polydisperse hard rods: The effect of fat tails in the length distribution
}

\author{
Alessandro Speranza, Peter Sollich \\ Department of Mathematics, King's College London, Strand, London WC2R 2LS, U.K. Email: peter.sollich@kcl.ac.uk
}

\begin{abstract}
We study the phase behaviour of hard rods with length polydispersity, treated within a simplified version of the Onsager model. We give a detailed description of the unusual phase behaviour of the system when the rod length distribution has a "fat" (e.g. log-normal) tail up to some finite cutoff. The relatively large number of long rods in the system strongly influences the phase behaviour: the isotropic cloud curve, which defines the point where a nematic phase first occurs as density is increased, exhibits a kink; at this point the properties of the coexisting nematic shadow phase change discontinuously. A narrow three-phase isotropic-nematic-nematic coexistence region exists near the kink in the cloud curve, even though the length distribution is unimodal. A theoretical derivation of the isotropic cloud curve and nematic shadow curve, in the limit of large cutoff, is also given. The two curves are shown to collapse onto each other in the limit. The coexisting isotropic and nematic phases are essentially identical, the only difference being that the nematic contains a larger number of the longest rods; the longer rods are also the only ones that show any significant nematic ordering. Numerical results for finite but large cutoff support the theoretical predictions for the asymptotic scaling of all quantities with the cutoff length.
\end{abstract}

\section{INTRODUCTION}

Rod-like particles in suspension can undergo a phase transition between an isotropic (I) phase without orientational order and a nematic $(\mathrm{N})$ phase where rods are preferentially oriented along the so-called nematic axis. This transition has been observed experimentally for chemical and biological systems ${ }^{1-5}$. Theoretically, the two main approaches for analysing systems of rod-like particles are due to Maier and Saupe ${ }^{6}$ and Onsager ${ }^{7}$. The Maier-Saupe theory is based on a long range attractive interaction between particles (originally conceived of as due to van der Waals forces) and neglects density variations. Such a theory is therefore appropriate for describing thermotropic liquid crystals, in which orientational phase transitions are induced by changes in temperature. The Onsager theory, on the other hand, only considers hard core (short range) interactions between particles. Temperature then becomes just a trivial factor setting the energy scale, and the Onsager theory therefore describes lyotropics: materials in which the phase transition is driven by a change in density of the system (at fixed temperature). The I-N phase transition is due, within the Onsager theory, to a competition between the orientational entropy - the tendency of rods to stay orientationally disordered - and the packing entropy; the latter is due to the excluded volume interaction, and higher for aligned particles. The key simplification of Onsager theory is to consider the "Onsager limit" of thin rods, where the ratio $D / L$ of the diameter and the length of the rods tends to zero. The free energy of the model can then be derived from a virial expansion truncated after the first nontrivial term, as all higher order terms turn out to be smaller by positive powers of $D / L$. To find the actual free energy for a given rod number density, one needs to minimize over the orientational distribution of the rods; once this is done, the I-N phase transition can be obtained by a standard double-tangent construction. Onsager solved the minimization problem by assuming a parametric form for the orientational distribution function; even with only one variational parameter, he obtained results for the densities of the coexisting phases ${ }^{7,8}$ which are in good agreement with the numerically exact solution obtained later ${ }^{9}$.

A comparison between experimental systems and the theoretical predictions of Onsager theory can be complicated due to e.g. non-hard interactions or particles that are not perfectly rigid $^{3,4,10,11}$. A further important factor is polydispersity, i.e. a spread in particle lengths and/or diameters ${ }^{12,5}$, and we focus in this paper on the effects that length polydispersity has on the phase behaviour of hard rods. Polydispersity makes the analysis of the problem more difficult, but also leads to much richer phase behaviour ${ }^{13}$. Even in simple biand tri-disperse systems, i.e. mixtures of rods of two or three different lengths, phase separation into two nematic $(\mathrm{N}-\mathrm{N})$ phases and three-phase I-N-N coexistence have been predicted theoretically ${ }^{12,8,14-16}$, and observed experimentally ${ }^{12}$. More generally, a pronounced broadening of the coexistence region and fractionation of the longer rods into the nematic phases are typical effects of length polydispersity ${ }^{12,17-19,5}$. These effects are also seen in simplified models, such as the polydisperse Zwanzig model $^{20,21}$ — where rods are only allowed to point along one of three orthogonal directions - or the lattice model developed by Flory et al. ${ }^{22-24}$. Within the context of the lattice model, exponential and Poisson ${ }^{25,26}$ as well as Gaussian $^{27}$ length distributions were analysed, with fractionation and a broadening of the coexistence region observed in all cases. The bidisperse case was also studied ${ }^{24}$ and, despite the rather different theoretical approach, showed phase behaviour qualitatively similar to that predicted within bidisperse Onsager theory ${ }^{28,29}$.

It is clear from the above discussion that more work 
is needed to understand the effects of length polydispersity on the phase behaviour of hard rods, especially with regard to the occurrence of the more "exotic" N-N and I-N-N phase coexistences. We recently began to investigate these questions, using the " $\mathcal{P}_{2}$ Onsager model" 30. This is obtained from the conventional Onsager theory by simplifying the angular dependence of the excluded volume term in the free energy, truncating a series expansion in Legendre polynomials after the first nontrivial term, which contains the second order Legendre polynomial $\mathcal{P}_{2}(\cos \theta)$. The simplicity of the resulting model enabled us to investigate in detail the phase behaviour resulting from different choices of rod length distributions. For a Schulz distribution - which is unimodal, i.e. has a single peak - fractionation and broadening of the coexistence region were found as expected, but neither $\mathrm{N}-\mathrm{N}$ nor I-N-N coexistence appeared. These more complex features did occur, however, for bidisperse and bimodal distributions; the latter were modelled by a mixture of two Schulz distributions peaked at different lengths. For the bidisperse case the appearance of these features is in encouraging qualitative agreement with the results of Onsager theory, suggesting that the approximation made in constructing the $\mathcal{P}_{2}$ model may not be crucial. A detailed analysis of the phase diagram of the $\mathcal{P}_{2}$ Onsager model showed that N-N and I-N-N regions only appeared if the ratio of the rod lengths was sufficiently large while the fraction of long rods in the system remained small. (For the bimodal case the individual peaks in the distribution also had to remain relatively narrow.) This suggested to us that N-N and I-N-N coexistence could also occur in unimodal length distributions that contain a larger number of long rods than the Schulz distribution with its exponentially decaying tail. We therefore investigate, in the present paper, the behaviour of the $\mathcal{P}_{2}$ Onsager model for rod length distributions with fat tails, i.e. decaying less than exponentially for large rod lengths. Our primary example will be the log-normal distribution; like the Schulz distribution, this has most of its weight around the average rod length, but contains a comparatively larger number of much longer rods; it has also yielded interesting results in previous work on lengthpolydisperse homopolymers ${ }^{31,32}$. Our main result is that I-N-N phase coexistence does indeed occur, although the topology of the phase diagram is rather different from the bidisperse case: the I-N-N region is very narrow in density and remains fully within the isotropic-nematic region, without being bordered by an N-N coexistence region.

The present paper is structured as follows. In Sec. II we give a brief description of the polydisperse $\mathcal{P}_{2}$ Onsager model and of the phase coexistence equations. We also motivate why the presence of fat tails should cause unusual phase behaviour. To make the phase coexistence problem well-defined, a cutoff on the rod length distribution needs to be introduced; this is of course also reasonable physically. Sec. III contains our numerical results for the phase diagram at finite cutoff, and we describe care- fully the unusual effects on phase behaviour caused by the presence of the long rods; it turns out that such effects are largely confined to a region of low densities where phase ordering would not yet be observed in systems with "conventional" length distributions. The second major part of the paper contains a theoretical treatment of the onset of nematic ordering, in the limit of large length cutoff (Sec. IV). This reveals many unusual features; in the limit of infinite cutoff, for example, both the densities and the rod volume fractions of the isotropic cloud phase and the coexisting nematic shadow phase coincide. The theoretical predictions are compared to the results of our numerical calculations, and show good agreement. We conclude in Sec. V with a summary and outlook towards future work. Technical material is relegated to two appendices.

\section{THE POLYDISPERSE $\mathcal{P}_{2}$ ONSAGER MODEL}

The $\mathcal{P}_{2}$ Onsager model is an approximate version of the Onsager model of hard rods. As in the Onsager theory, particles are modelled as rigid spherocylinders with hard core interaction. Phase transitions are driven by density rather than temperature; the latter simply fixes the energy scale and can be set to unity ${ }^{7,21,20,30}$.

We allow for length polydispersity, with the rod lengths $L$ distributed according to a length distribution $P(L)$, while assuming that all rod diameters $D$ are equal. To be able to take the Onsager limit of thin rods simultaneously for all rod lengths in $P(L)$, we introduce a reference length $L_{0}$ and consider the limit $D / L_{0} \rightarrow 0$ while keeping the normalized lengths $l=L / L_{0}$ constant. The thermodynamic state of the system is specified by the density distribution $\rho(l, \theta)$, where $\rho(l, \theta) d l d \Omega /(4 \pi)$ gives the number density of rods with (normalized) lengths in the range $l \ldots l+d l$ and orientations in a solid angle $d \Omega$ around any direction at an angle $\theta$ with the nematic $\operatorname{axis}^{30,20}$ We can then decompose $\rho(l, \theta)$ into

$$
\rho(l, \theta)=\rho(l) P_{l}(\theta)=\rho_{0} P(l) P_{l}(\theta)
$$

where we have isolated the overall density $\rho_{0}$ and the normalized length distribution $P(l)$. The angular probability distributions $P_{l}(\theta)$ are normalized in such a way that

$$
\int \widetilde{d \theta} P_{l}(\theta)=1
$$

using the shorthand

$$
\widetilde{d \theta}=\frac{1}{2} d \cos \theta
$$

and the convention that all angular integrations are over the range $0 \ldots \pi$. Conversely, the density $\rho_{0}$ is obtained by integrating $\rho(l, \theta)$ over all $l$ and $\theta$,

$$
\rho_{0}=\int d l \widetilde{d \theta} \rho(l, \theta)=\int d l \rho(l)
$$


The excess free energy of the model arises from the excluded volume interaction of the rods. In the Onsager limit, the virial expansion can be truncated after the second-order term. The simplification of the $\mathcal{P}_{2}$ Onsager model consists in simplifying the angular dependence of the resulting excluded volume term, by expanding in Legendre polynomials ${ }^{9}$ and truncating after the first nontrivial term. If all densities are measured in units of $1 /\left[(\pi / 4) D L_{0}^{2}\right]$, the excess free energy density then becomes simply ${ }^{30}$

$$
\tilde{f}=\frac{c_{1}}{2} \rho_{1}^{2}-\frac{c_{2}}{2} \rho_{2}^{2}
$$

where $c_{1}=2$ and $c_{2}=5 / 4$. We have also defined the two moments of the density distribution

$$
\rho_{1}=\int d l \widetilde{d \theta} l \rho(l, \theta)=\int d l l \rho(l) \int \widetilde{d \theta} P_{l}(\theta)
$$

and

$\rho_{2}=\int d l \widetilde{d \theta} l \mathcal{P}_{2}(\cos \theta)=\int d l l \rho(l) \int \widetilde{d \theta} \mathcal{P}_{2}(\cos \theta) P_{l}(\theta)$

The first of these is just the rescaled rod volume fraction, $\rho_{1}=\left(L_{0} / D\right) \phi$, while $\rho_{2}$ contains information on the orientational order in the system. It is clear from Eq. (5) that for an isotropic phase $\left(P_{l}(\theta) \equiv 1\right) \rho_{2}$ vanishes, while $0<\rho_{2} \leq \rho_{1}$ for a nematic phase, whose angular distribution will be peaked around $\theta=0$ and $\theta=\pi ; \rho_{2}$ is therefore a natural orientational order parameter for the system. In fact one can rewrite $\rho_{2}$ as

$$
\rho_{2}=\int d l \widetilde{d \theta} l \mathcal{P}_{2}(\cos \theta) \rho(l) P_{l}(\theta)=\int d l l \rho(l) S(l)
$$

if one defines the order parameter $S(l)$ as the average of the second Legendre polynomial for rods of given length,

$$
S(l)=\int \widetilde{d \theta} \mathcal{P}_{2}(\cos \theta) P_{l}(\theta)
$$

Eq. (6) can also be read as $\rho_{2}=\rho_{0}\langle l S(l)\rangle$, where the average is taken over the normalized length distribution $P(l)=\rho(l) / \rho_{0}$.

As usual the ideal part of the free energy, comprising the ideal gas term and the entropy of mixing, is

$$
f_{\mathrm{id}}=\int \widetilde{d \theta} d l \rho(l, \theta)[\ln \rho(l, \theta)-1]
$$

Using the decomposition (1) and the normalization (2) we can then write the total free energy as

$f=\int d l \rho(l)[\ln \rho(l)-1]+\int d l \rho(l) \int \widetilde{d \theta} P_{l}(\theta) \ln P_{l}(\theta)+\tilde{f}$

with $\tilde{f}$ given by Eq. (3).

\section{A. Phase coexistence equations}

Since the rod orientations - as opposed to their lengths - are not conserved, the orientational probability distributions $P_{l}(\theta)$ for a given density distribution $\rho(l)$ over rod lengths have to be found by minimizing the free energy (9), subject to the constraints (2). Introducing appropriate Lagrange multipliers $\kappa(l)$ we obtain the condition

$$
\begin{array}{r}
\frac{\delta}{\delta P_{l}(\theta)}\left(f+\int d l \kappa(l) \int \widetilde{d \theta} P_{l}(\theta)\right)= \\
\rho(l)\left[\ln P_{l}(\theta)+1\right]+l \rho(l)\left[c_{1} \rho_{1}-c_{2} \rho_{2} \mathcal{P}_{2}\right]+\kappa(l)=0
\end{array}
$$

Solving for $P_{l}(\theta)$ gives

$$
P_{l}(\theta)=\frac{\exp \left(l c_{2} \rho_{2} \mathcal{P}_{2}\right)}{\int \widetilde{d \theta} \exp \left(l c_{2} \rho_{2} \mathcal{P}_{2}\right)}
$$

where we have introduced the shorthand $\mathcal{P}_{2}=\mathcal{P}_{2}(\cos \theta)$. This, together with (5) gives a self-consistency equation

$$
\rho_{2}=\int d l l \rho(l) \frac{\int \widetilde{d \theta} \mathcal{P}_{2} \exp \left(l c_{2} \rho_{2} \mathcal{P}_{2}\right)}{\int \widetilde{d \theta} \exp \left(l c_{2} \rho_{2} \mathcal{P}_{2}\right)}
$$

which can be solved for $\rho_{2}$.

To calculate phase coexistences, we need the expression for the chemical potentials and for the osmotic pressure. The chemical potentials are obtained by taking a derivative of the free energy with respect to the density distribution $\rho(l)$,

$$
\begin{aligned}
\mu(l) & =\frac{\delta f}{\delta \rho(l)} \\
& =\ln \rho(l)-\ln \int \widetilde{d \theta} \exp \left(-l c_{1} \rho_{1}+l c_{2} \rho_{2} \mathcal{P}_{2}\right)
\end{aligned}
$$

where we do not need to differentiate explicitly with respect to the $P_{l}(\theta)$ since they are chosen to minimize the free energy. The osmotic pressure, derived from the Gibbs-Duhem relation, is

$$
\Pi=-f+\int d l \rho(l) \mu(l)=\rho_{0}+\frac{c_{1}}{2} \rho_{1}^{2}-\frac{c_{2}}{2} \rho_{2}^{2}
$$

Equality of the chemical potentials in a set of coexisting phases labelled by $a=1 \ldots P$ then leads to the following expression for the length distribution

$$
\rho^{(a)}(l)=R(l) \int \widetilde{d \theta} \exp \left(-l c_{1} \rho_{1}^{(a)}+l c_{2} \rho_{2}^{(a)} \mathcal{P}_{2}\right)
$$

where $R(l)$ is a function common to all phases. It can be obtained by imposing the lever rule or particle number conservation,

$$
\sum_{a} v^{(a)} \rho^{(a)}(l)=\rho^{(0)}(l)
$$


where $\rho^{(0)}(l)$ is the overall or 'parent' density distribution of the system and $v^{(a)}$ is the fraction of the system volume occupied by phase $a$. This gives

$$
R(l)=\frac{\rho^{(0)}(l)}{\sum_{a} v^{(a)} \int \widetilde{d \theta} \exp \left(-l c_{1} \rho_{1}^{(a)}+l c_{2} \rho_{2}^{(a)} \mathcal{P}_{2}\right)}
$$

Using Eqs. $(10,14)$, the full density distributions over lengths and orientations are therefore

$$
\rho^{(a)}(l, \theta)=\frac{\rho^{(0)}(l) \exp \left(-l c_{1} \rho_{1}^{(a)}+l c_{2} \rho_{2}^{(a)} \mathcal{P}_{2}\right)}{\sum_{b} v^{(b)} \int \widetilde{d \theta^{\prime}} \exp \left(-l c_{1} \rho_{1}^{(b)}+l c_{2} \rho_{2}^{(b)} \mathcal{P}_{2}^{\prime}\right)}
$$

We thus have a closed system of equations whose solutions determine the phase behaviour of the $\mathcal{P}_{2}$ Onsager model. For each of the $P$ phases we have 3 unknowns, $\rho_{1}^{(a)}, \rho_{2}^{(a)}$ and $v^{(a)}$, giving $3 P$ unknowns in total. For each phase, $\rho_{1}^{(a)}$ and $\rho_{2}^{(a)}$ obey the equations obtained by substituting Eq. (17) into Eqs. (4,5); for $\rho_{2}^{(a)}$ this leads back to Eq. (11). The remaining $P$ equations are given by the equality of the osmotic pressure in all phases $(P-1$ equations) and the normalization condition $\sum_{a} v^{(a)}=1$. The lever rule and the equality of chemical potentials are already ensured by having density distributions in the different phases of the form (17).

So far everything holds for arbitrary parent distributions $\rho^{(0)}(l)$. We will denote by $\rho_{0}^{(0)}$ the overall parent number density, so that $P^{(0)}(l)=\rho^{(0)}(l) / \rho_{0}^{(0)}$ is the normalized parent rod length distribution. We always assume that the average rod length in the parent is one; a different value could be absorbed into the reference length $L_{0}$.

\section{B. Fat-tailed distributions}

We now motivate why the phase behaviour of systems with rod length distributions with fat (less than exponentially decaying) tails should be unusual. To do so, it is useful to focus on the onset of the isotropic-nematic coexistence (the so called "isotropic cloud point"). Equality of the chemical potentials (12) between an isotropic $\left(\rho^{\mathrm{I}}(l)\right)$ and a nematic $\left(\rho^{\mathrm{N}}(l)\right)$ phase gives

$$
\rho^{\mathrm{N}}(l)=\rho^{\mathrm{I}}(l) e^{\beta l} \int \widetilde{d \theta} \exp \left[c_{2} \rho_{2}^{\mathrm{N}} l\left(\mathcal{P}_{2}-1\right)\right]
$$

where we have defined

$$
\beta=-c_{1}\left(\rho_{1}^{\mathrm{N}}-\rho_{1}^{\mathrm{I}}\right)+c_{2} \rho_{2}^{\mathrm{N}}
$$

and used $\rho_{2}^{\mathrm{I}}=0$. At the isotropic cloud point, i.e. at the onset of the phase coexistence between the isotropic parent and an infinitesimal amount of nematic "shadow" phase, $\rho^{\mathrm{I}}(l)=\rho^{(0)}(l)$. Moreover, $\beta$ should be positive since the nematic phase will have the larger rod volume fraction, $\rho_{1}^{\mathrm{N}}>\rho_{1}^{\mathrm{I}}$. The angular integral in Eq. (18), finally, is bounded by unity since $\mathcal{P}_{2}-1 \leq 0$, and a Gaussian approximation around $\theta=0$ that is valid for large $l$ shows that it varies only as a power law with $l$. Taken together, these facts imply that as soon as the parent distribution $\rho^{(0)}(l)$ is less than exponentially decaying with $l$, the nematic length distribution would diverge for large $l$. In order to ensure convergent integrals for the density $\rho_{0}^{\mathrm{N}}$ and the rod volume fraction $\rho_{1}^{\mathrm{N}}$ we will therefore need to impose a maximum length cutoff $l_{m}$ on the parent distribution. The need for such a cutoff suggests - and we will find this confirmed below - that even though there are only a very small number of long rods (since the integral $\int d l \rho^{(0)}(l)$ converges) they can dominate the onset of phase coexistence from the isotropic side. Notice that, coming from the nematic, no such effects are expected. At the nematic cloud point, $\rho^{\mathrm{N}}(l)=\rho^{(0)}(l)$. Inverting Eq. (18) one sees then that the length distribution in the isotropic shadow phase is well behaved even for $l_{m} \rightarrow \infty$, as $\beta$ is still positive and the angular integral is harmless.

Motivated by these insights, we consider fat-tailed parent distributions in the rest of this paper. We expect, and indeed find, that the presence of the fat tail, and the value of the cutoff $l_{m}$, will have a strong influence on the isotropic cloud point and the corresponding nematic shadow, while leaving the nematic cloud point essentially unaffected. Although many of our results are valid for general fat-tailed distributions, we will normally focus on log-normal distributions for definiteness.

\section{NUMERICAL RESULTS}

In this section we describe the unusual phase behaviour of systems with a log-normal parent length distribution

$$
P^{(0)}(l)=\frac{1}{\sqrt{2 \pi w^{2}} l} \exp \left[-\frac{(\ln l-\mu)^{2}}{2 w^{2}}\right]
$$

with a finite cutoff, $l \leq l_{m}$. The parameters $\mu$ and $w$ are chosen such that the average rod length is $\langle l\rangle=1$ and the polydispersity $\sigma$, i.e. the normalized standard deviation of distribution of rod lengths defined through

$$
\sigma^{2}=\frac{\left\langle l^{2}\right\rangle}{\langle l\rangle^{2}}-1
$$

has the desired value; explicitly, this gives $w^{2}=\ln \left(1+\sigma^{2}\right)$ and $\mu=-w^{2} / 2$. Note that for finite cutoff $l_{m}, P^{(0)}(l)$ as given will not be normalized precisely to one, and $\langle l\rangle$ and $\left\langle l^{2}\right\rangle$ will differ slightly from the desired values of 1 and $1+\sigma^{2}$. However, even for modest cutoffs the deviations are very small. For instance, at cutoff $l_{m}=50$ and $\sigma=$ 0.5 , the integrals $\int d l P^{(0)}(l) l^{n}$ differ from their $l_{m} \rightarrow \infty$ values 1,1 and $1+\sigma^{2}$ by values of order $10^{-17}, 10^{-15}$, and $10^{-13}$. In the following we will neglect these small corrections.

Due to the numerical difficulties introduced by polydispersity, and compounded by the presence of a fat 
tail in the parent distribution, we used the moment method $^{33-35}$ to solve numerically an approximate system of phase coexistence equations, applying the recently developed adaptive method ${ }^{20,30}$ to keep deviations from the exact solution as small as possible. We found that controlling such deviations was rather more difficult than for more "well-behaved" rod length distributions, and therefore decided to integrate the moment method with the exact solution. In this mixed method, we effectively use the adaptive moment method to produce a good starting point for the numerical solution of the exact phase coexistence conditions. This method allows us to calculate the exact phase behaviour, while avoiding the convergence problems that result from a direct numerical attack on the exact phase coexistence conditions.

\section{A. Overview of the limiting theory}

Before showing the numerical results at finite cutoff $l_{m}$, it is useful to anticipate some of the analytical results which we will derive later on, in Sec. IV, for the large cutoff limit. This helps to understand and physically interpret some of the unusual features of the phase diagram obtained at finite cutoff.

The main feature affecting the physics of the system is obviously the presence of a large (relative to more strongly decaying distributions, e.g. of Schulz type) number of long rods. Because of the weighting with $l$ in the moment densities $(4,5)$ appearing in the excess free energy, it is plausible that these long rods can drive the phase ordering. Indeed, our theory will show that rods with $l$ of the order of the cutoff $l_{m}$ become strongly ordered already when the nematic phase first appears, and that this ordering drives the onset of phase coexistence to lower densities. The isotropic cloud point therefore depends on the cutoff, moving to lower densities as $l_{m}$ increases. However, the ordering of the longest rods is not sufficient to precipitate general order in the nematic phase. In fact, we find that at the relatively low isotropic cloud point densities caused by the presence of the long rods, the short rods with $l$ of order unity are not yet able to order, staying completely disordered in the limit $l_{m} \rightarrow \infty$. The system thus has an unusual initial phase separation, in which the nematic phase is distinguishable from the isotropic phase only because it contains a somewhat larger fraction of the longest rods, which are also nematically ordered; for the short rods, the density distribution is essentially identical in both phases. At finite $l_{m}$ the contribution of the longest rods causes the nematic phase to have a larger rod volume fraction and average rod length than the isotropic phase. As $l_{m}$ increases, however, the differences turn out to vanish, and the number density and rod volume fraction of the nematic shadow phase become identical to those of the isotropic cloud phase. In a plot of these quantities versus the polydispersity $\sigma$, the resulting cloud and shadow curves therefore coincide for $l_{m} \rightarrow \infty$. In fact, we will obtain this limiting curve explicitly as

$$
\rho_{0}=\rho_{1}=\frac{1}{4} \frac{1}{\left(\sigma^{2}+1\right)}
$$

Our theory also predicts the leading terms in the approach to this asymptotic behaviour as $l_{m}$ increases. The number density and volume fraction of the isotropic and the nematic phase turn out to differ, in the limit, by terms proportional to $\rho_{2}^{\mathrm{N}}$; here $\rho_{2}^{\mathrm{N}}$ is the value of the moment density (5) in the nematic phase, which for a lognormal parent scales as $\left(\ln l_{m}\right) / l_{m}^{1 / 2}$. The parameter $\beta$ in Eq. (18) turns out to scale like $\left(\rho_{2}^{\mathrm{N}}\right)^{2}$ to leading order. This decrease of $\beta$ to zero allows the theory to remain well-defined, with finite values for the number density and rod volume fraction of the nematic for $l_{m} \rightarrow \infty$. In fact, the scaling of $\beta$ is such that the two factors $\exp (\beta l)$ and $\rho^{\mathrm{I}}(l)=\rho^{(0)}(l)$ in Eq. (18) approximately cancel for $l=l_{m}$, with their product varying only as a power law in $l_{m}$. In our numerical results at finite $l_{m}$, this feature will be visible as a peak at $l=l_{m}$ in the density distribution of the nematic shadow phase. The power law scaling of the weight of the peak is such that its contribution to the moment densities $\rho_{0}^{\mathrm{N}}$ and $\rho_{1}^{\mathrm{N}}$ becomes negligible for $l_{m} \rightarrow \infty$, as pointed out above. On the other hand, higher moments of the nematic density distribution, $\int d l \rho^{\mathrm{N}}(l) l^{n}$ with $n \geq 2$, are dominated by the contribution from the peak and actually diverge with $l_{m}$. Similar unusual features also appear in, for example, the Flory-Huggins theory of polymers with log-normal chain length distribution ${ }^{31,32}$.

\section{B. Overall phase diagram topology}

In Fig. 1 we show the phase diagram obtained for a lognormal parent distribution with cutoff $l_{m}=100$. Plotted is the density of the parent at which phase transitions occur; the lines separating one- and two-phase regions are therefore cloud curves.

Vertically, the phase diagram is broadly divided into two regions: in the first one, at low polydispersity, the phase behaviour mirrors closely that of a system with a Schulz distribution of rod lengths ${ }^{30}$, exhibiting an I$\mathrm{N}$ coexistence region in the middle and single-phase I and $\mathrm{N}$ regions to the left and right. The isotropic cloud curve essentially coincides, at these small values of $\sigma$, with the one found for a Schulz length distribution ${ }^{30}$ and indicated by the dashed line in Fig. 1. At the other end of the I-N coexistence region the nematic cloud curve (not shown) is, as expected from the discussion in Sec. II B, essentially unaffected by the presence of long rods and remains close to its analogue for a Schulz distribution in the whole range of $\sigma$.

The second region of the phase diagram, at higher $\sigma$, is more strongly affected by the relatively large number of long rods in the log-normal parent distribution, and 


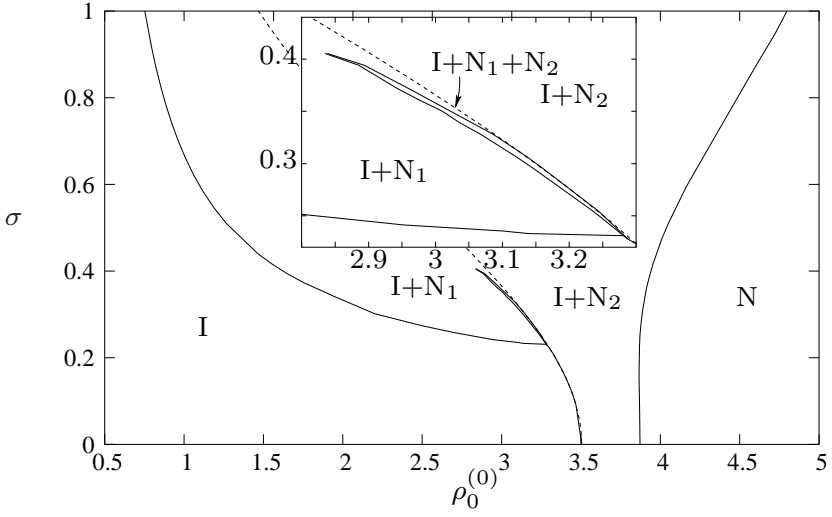

FIG. 1. Phase diagram for a log-normal parent with cutoff $l_{m}=100$. Shown is the polydispersity $\sigma$ plotted against the density of the parent $\rho_{0}^{(0)}$ at which phase transitions occur. From left to right we find a single-phase isotropic phase, the I-N coexistence region and then the single-phase nematic; the coexistence region is bounded from left and right by the isotropic and nematic cloud curves, respectively. Inside the I-N coexistence region we observe a narrow three-phase I-N-N region. This is shown in more detail in the inset and lies close to the isotropic cloud curve (dashed line) that would result for a Schulz distribution. Above the three-phase region one has, instead of N-N phase separation, a crossover from a cutoff-dependent regime (located to the right of the isotropic cloud curve) to a regime in which the phase behaviour is almost unaffected by $l_{m}$; the location of the crossover is close to the dashed line, i.e. the "conventional" (Schulz) cloud curve.

therefore also by the value of the cutoff $l_{m}$. At the boundary between the two regions, at $\sigma \approx 0.24$, the cloud curve (see Fig.1) has a kink. Correspondingly, the properties of the coexisting nematic shadow phases change discontinuously at this point, from a "conventional" nematic phase similar to that found for a Schulz distribution to one containing more, and more strongly ordered, long rods; see Figs. 2 to 4 below. Intuitively, below the threshold value of $\sigma$ the parent distribution is narrow enough to keep the number of long rods small, to the point where phase behaviour is not significantly affected, while above the threshold the influence of these long rods can become dominant.

Precisely at the kink in the isotropic cloud curve, the isotropic phase coexists with two different shadow nematic phases. As expected, we therefore find a threephase region beginning at this point. Crossing the isotropic cloud curve from the left, the system thus first separates into an isotropic phase and an "unusual" nematic $\left(\mathrm{N}_{1}\right)$ with many long rods. Upon increasing density, there is a narrow interval of three-phase $\mathrm{I}-\mathrm{N}_{1}-\mathrm{N}_{2}$ coexistence, with $\mathrm{N}_{2}$ being a "conventional" nematic phase. Beyond this point, the system behaves in the usual way, showing an $\mathrm{I}-\mathrm{N}_{2}$ coexistence and finally a single nematic phase. On increasing $\sigma$, however, the three-phase region eventually closes off and the strict transition between $\mathrm{N}_{1}$ and $\mathrm{N}_{2}$ is replaced by a pronounced crossover in the shape of the density distribution of the nematic phase; see Sec. III D below. The crossover line in the phase diagram essentially continues the three-phase region, and both are close to the isotropic cloud curve for a Schulz distribution (dashed line in Fig. 1), which is representative also of other parent distributions without fat tails. We can thus say that it is essentially only to the left of this line in the phase diagram that the fat tail of the lognormal parent, and therefore also the value of the cutoff, significantly affect phase behaviour; one of the resulting effects is of course that, above the threshold value of $\sigma$, the actual isotropic cloud curve is moved significantly to the left of its counterpart for a Schulz distribution. The unaffected region of the phase diagram includes, in particular, the nematic cloud curve as expected from the discussion in Sec. II B.

The fact that the three-phase region terminates at a finite value of $\sigma$, where a strict phase coexistence involving two nematic phases turns into a crossover in the properties of a single nematic phase, indicates that there must be a nematic-nematic critical point on the I-N-N phase boundary. However, because the three-phase region itself is extremely narrow, we have not been able to locate this point numerically.

\section{Cloud and shadow curves}

We now discuss in more detail the effect of the long rods on the isotropic cloud and nematic shadow curves; as shown above, the nematic cloud and isotropic shadow curves do not need to be considered further since they are essentially unaffected by the presence of the long rods. Fig. 2 shows the isotropic cloud (main plot) and nematic shadow (inset) curves, in the $\rho_{0}-\sigma$ representation of plotting polydispersity versus the number densities $\rho_{0}$ in the cloud and shadow phases. The kink in the cloud curves can clearly be seen, as can the associated discontinuity in the shadow curves. Upon increasing the cutoff length, the curves also move towards the predicted limit curve for $l_{m} \rightarrow \infty$. (We defer a discussion of the numerical results for the actual rate of convergence to Sec. IV F.) The same is true if, as shown in Fig. 3, we use the rescaled volume fractions $\rho_{1}$ rather than the number densities to represent the properties of the cloud and shadow phases. Interestingly, at the discontinuity in the shadow curves the volume fraction $\rho_{1}^{\mathrm{N}}$ of the nematic phase becomes larger (Fig. 3) as $\sigma$ is increased, while the number density $\rho_{0}^{\mathrm{N}}$ becomes much smaller and drops below that of its isotropic counterpart (Fig. 2). This implies an increase in the average rod length $\langle l\rangle=\rho_{1}^{\mathrm{N}} / \rho_{0}^{\mathrm{N}}$ of the nematic phase, i.e. a strong fractionation effect which is rather more pronounced than for a system with a Schulz distribution. This is shown explicitly in Fig.4, where we plot the average rod length in the nematic shadow phases against the 


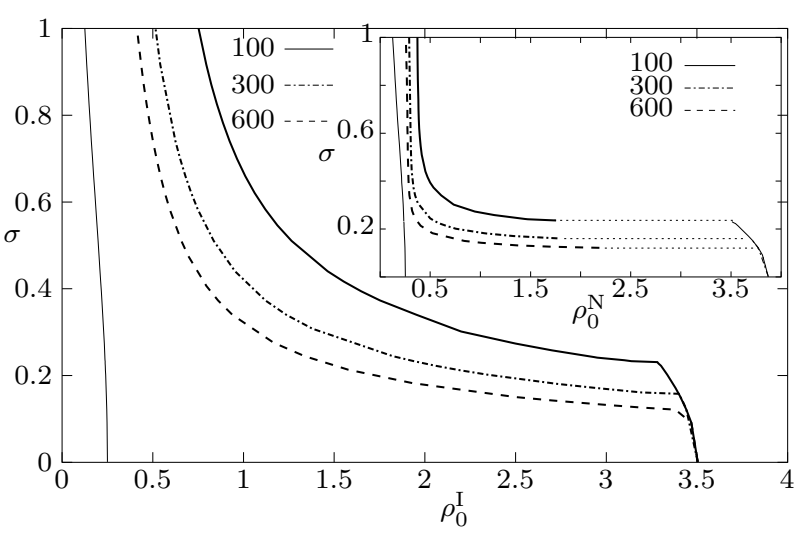

FIG. 2. Isotropic cloud curve and nematic shadow curve (in the inset) in the $\rho_{0}-\sigma$ representation, for the different values of the cutoff $l_{m}$ shown in the legend. Even for the modest values of $l_{m}$ used, the evolution of the curves with increasing $l_{m}$ is compatible with the predicted convergence to the same limiting curve (thin solid line) for both isotropic cloud and nematic shadow. Note the discontinuity in each shadow curve, which corresponds to the kink in the associated cloud curve; here the nematic phase jumps from a "normal" phase to one dominated by the long rods. Below the kink or discontinuity, the cloud and shadow curves are practically cutoff-independent and essentially identical to those found for a Schulz distribution.

polydispersity. The discontinuities in the shadow curves for $\rho_{1}$ and $\rho_{0}$ give a similar discontinuity here; below it, the results are very close to those for a Schulz distribution, while for higher values of $\sigma$ the rod length in the nematic shadow phase is considerably larger, indicating that the nematic phase has become enriched in the long rods. (Note that the coexisting isotropic cloud phase always has average rod length one, since its density distribution is identical to that of the parent.) As $\sigma$ is increased, the fractionation first increases, but then reaches a maximum and decreases again. This can be understood by looking back at Figs. 2 and 3: the nematic shadow's number density $\rho_{0}^{\mathrm{N}}$ initially decreases more quickly with increasing $\sigma$ than its rod volume fraction $\rho_{1}^{\mathrm{N}}$, leading to the observed increase in $\langle l\rangle=\rho_{1}^{\mathrm{N}} / \rho_{0}^{\mathrm{N}}$; but eventually $\rho_{0}^{\mathrm{N}}$ becomes almost independent of $\sigma$ and $\langle l\rangle$ then decreases with $\rho_{1}^{\mathrm{N}}$. It is intriguing that the maximum fractionation is reached at roughly the same value of $\sigma$ at which the three-phase region disappears (compare Fig. 1), but we have no explanation for this at present.

For completeness, Fig. 4 also shows the average rod length in the isotropic shadow; recall that this is defined as the isotropic phase which coexists with the nematic cloud phase at the upper limit (in density) of the phase separation region. As expected, the results show no pronounced effect from the presence of long rods and are close to those for a Schulz distribution.

More insight into the properties of the isotropic cloud and nematic shadow phases can be gained by plotting

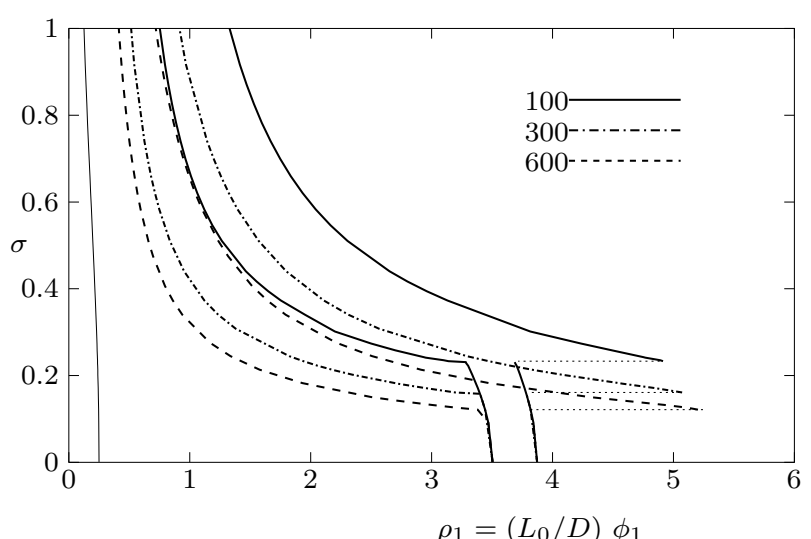

FIG. 3. Analogue of Fig. 2, but in the $\rho_{1}-\sigma$ representation, i.e. with (rescaled) volume fractions $\rho_{1}$ used instead of number densities $\rho_{0}$ to indicate the properties of the phases. Note the large increase in $\rho_{1}^{\mathrm{N}}$ at the discontinuity of the nematic shadow curves, which contrasts with a decrease in $\rho_{0}^{\mathrm{N}}$ (see Fig. 2). Below the discontinuity, the results are again very similar to those for a Schulz distribution.

their density distributions $\rho(l)$. Fig. 5 shows exemplary results for $l_{m}=100$ and a value of $\sigma$ above the discontinuity in the shadow curve, i.e. in the region of the phase diagram where the long rods have a significant effect. As anticipated in Sec. III A, the density distribution of the nematic shadow for rod lengths of order unity has a shape similar to that of the isotropic cloud phase, while exhibiting an exponential increase for rod lengths $l$ comparable to the cutoff $l_{m}$. Note that the total density in this long rods part of the distribution is much smaller (by almost two orders of magnitude in the example) than the overall nematic density; but the fact that the lengths themselves are large makes this sufficient to give the large average rod lengths $\langle l\rangle$ that we found above. With increasing cutoff the peak moves to larger lengths while, as we will see later in more detail, its weight decreases. This is why, in the limit $l_{m} \rightarrow \infty$, the isotropic cloud and nematic shadow phase become indistinguishable in their number density and rod volume fraction, i.e. in their moments $\rho_{0}$ and $\rho_{1}$; as a consequence, the average rod length in the nematic shadow phase also tends to unity (the value in the parent) in the limit.

Looking ahead to our theoretical treatment below, the fact that the nematic density distribution has two maxima, one for $l$ of order unity and a small second peak for $l=l_{m}$, will allow us to split integrals over rod lengths into corresponding short and long rod parts. The logscale representation of the density distribution in the nematic cloud phase (Fig. 5, bottom) supports the viability of this approach, showing a clear $\operatorname{dip}$ of $\rho^{\mathrm{N}}(l)$ to negligible values between the short and long rod regimes. The fact that the decay from the second peak in $\rho^{\mathrm{N}}(l)$, for $l<l_{m}$, is close to exponential will further simplify matters, allowing us to replace non-exponential factors in the long 


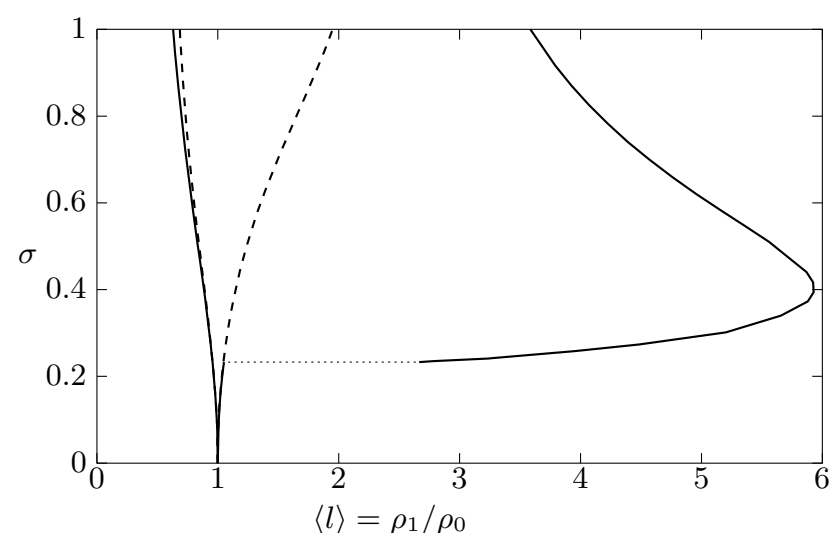

FIG. 4. The average rod length $\langle l\rangle$ in the nematic and isotropic shadow phases (solid) at cutoff $l_{m}=100$, compared with the same results for a system with a Schulz distribution (dashed). The isotropic shadow, i.e. the phase which initially coexists with the nematic when the density is reduced from large values, is in both cases the one at lower $\langle l\rangle$; as expected, it is essentially unaffected by the presence of long rods so that the results for the log-normal and the Schulz distributions are very similar. The same is true for the nematic shadow phase below the threshold value of $\sigma$; above, the average rod length is much larger. The nematic phase here has an enhanced concentration of the longest rods, leading to pronounced fractionation.

rod integrals by their values $l=l_{m}$.

Finally, we comment on the strength of orientational order in the nematic cloud phase. In Sec. III A we anticipated that in the limit of large cutoff this actually vanishes, as indicated by the convergence of $\rho_{2}^{\mathrm{N}}$ to zero. This implies that rods with lengths $l$ of order unity are, in the limit, orientationally disordered: Eq. (10) gives a uniform orientational distribution when $\rho_{2}^{\mathrm{N}} l \rightarrow 0$. More specifically, Eq. (10) implies that only rods with $l$ of or$\operatorname{der} 1 / \rho_{2}^{\mathrm{N}}$ and greater show significant orientational order, and $\rho_{2}^{\mathrm{N}} \rightarrow 0$ for $l_{m} \rightarrow \infty$ implies that this "ordering length" $1 / \rho_{2}^{N}$ diverges (while remaining $\ll l_{m}$; see Sec. IV D). The convergence of $\rho_{2}^{N}$ to zero is rather slow however, and for the modest values of $l_{m}$ used so far $\rho_{2}^{N}$ is still of order unity. For example, the nematic shadow phase at $\sigma=0.5$ and $l_{m}=100$ has $\rho_{2}^{\mathrm{N}} \approx 2$ and therefore noticeable orientational order even for rod lengths $l$ of order one.

\section{Long rod effects in the coexistence region}

In Sec. III B, we reported that the region of the phase diagram most strongly affected by the long rods is located above the treshold value of $\sigma$ and between the actual isotropic cloud curve and that which is obtained for distributions without fat tails, e.g. of Schulz type. We now explore this region in more detail, focussing on the regime of large $\sigma$ where there is no three-phase I-N-N co-
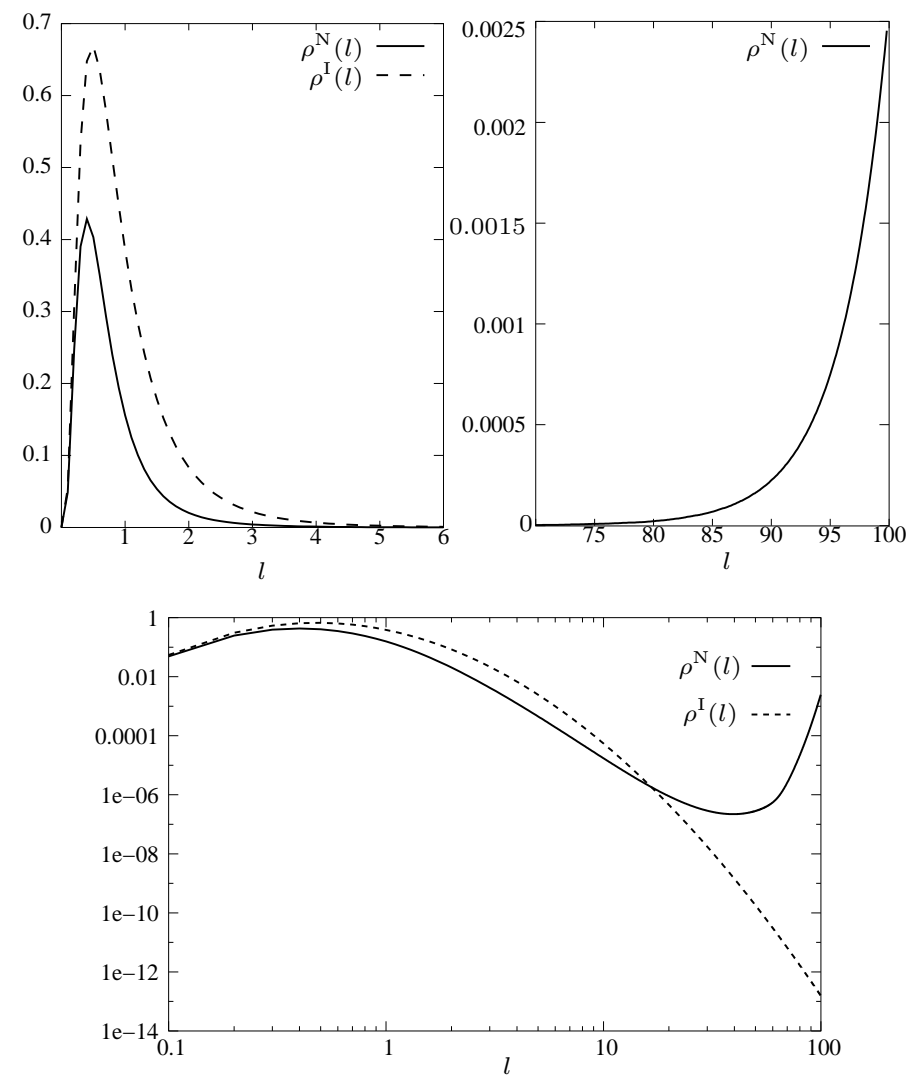

FIG. 5. Density distribution of the isotropic cloud phase (solid) and the nematic shadow phase (dashed), at the isotropic cloud point for $l_{m}=100$ and $\sigma=0.63$, in linear (top left and right) and log-log (bottom) scale. The top right panel shows the region of large $l$, where the nematic shadow distribution exhibits an exponentially increasing regime. The bottom panel demonstrates that, given the very small density of such long rods in the isotropic phase, their enrichment in the nematic phase is quite dramatic.

existence but instead a pronounced change in the properties of the nematic phase as the coexistence region is traversed.

While at the isotropic cloud point it is only the longer rods in the nematic phase that exhibit noticeable orientational order (at least for large cutoff $l_{m}$; see end of previous section), we expect also the shorter rods to become ordered eventually as density is increased. As the effect of the longest rods on the nematic phase diminishes, fractionation effects are then expected to become less pronounced, leading to a reduced average rod length in the nematic. A plot of the average rod lengths in the isotropic and nematic phases against the parent density across the coexistence region (Fig. 6) shows this effect clearly. At parent densities $\rho_{0}^{(0)} \approx 2$ we see a crossover, where the average rod length in the nematic drops to the values typical for phase separation from a parent distribution without a fat tail; as expected, the crossover den- 


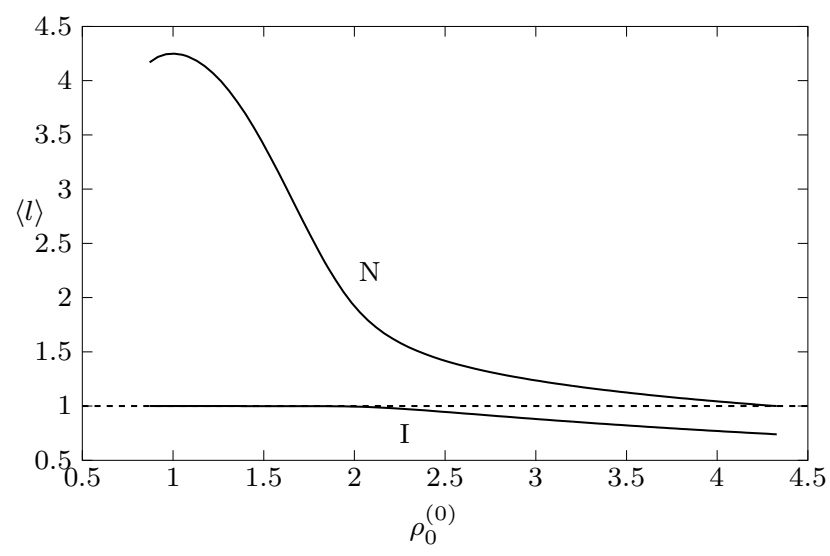

FIG. 6. Average rod length in the isotropic and nematic phase across the coexistence region at $\sigma=0.7$ and $l_{m}=100$. Notice the strong fractionation in the first, "long rod-dominated", part of the coexistence region up to parent densities around $\rho_{0}^{(0)}=2$, while above the behaviour resembles that for a parent with e.g. a Schulz distribution. Notice also the non-monotonicity of the average rod length in the nematic phase, which is consistent with our theory for the large-cutoff limit (see text).

sity is close to the isotropic cloud point $\left(\rho_{0}^{(0)} \simeq 2.1\right)$ for a Schulz distribution with the same polydispersity. Notice that the average rod length in the nematic phase, $\langle l\rangle^{\mathrm{N}}$, is non-monotonic in parent density, initially showing an increase when moving away from the isotropic cloud point but then decreasing again. This is consistent with our theory for the large cutoff-limit, which predicts that for $l_{m} \rightarrow \infty,\langle l\rangle^{\mathrm{N}}=\langle l\rangle^{\mathrm{I}}(=1$ with our convention for the parent's average rod length) at the isotropic cloud point. On increasing the density, $\langle l\rangle^{\mathrm{N}}$ must then first increase as the system begins to fractionate, before reducing again to unity as the nematic cloud point is approached.

The variation of the density of the two phases across the coexistence region (Fig. 7) similarly shows the crossover between two different regimes for the nematic phase: in the low-density regime, the density of the nematic increases rather quickly with the parent density, before crossing over to the more gradual variation that is familiar from systems with length distributions without fat tails. Interestingly, the density of the isotropic phase is essentially identical to that of the parent throughout the low-density region where the phase separation behaviour is dominated by the long rods. Since the overall density of the system must equal that of the parent, the fractional system volume occupied by the nematic phase must then be very small. This is indeed what we find, and the representation in Fig. 8 of the phase behaviour provides probably the clearest visual demonstration of the crossover between the low-density regime dominated by long rods and the region of more conventional phase behaviour at higher densities.

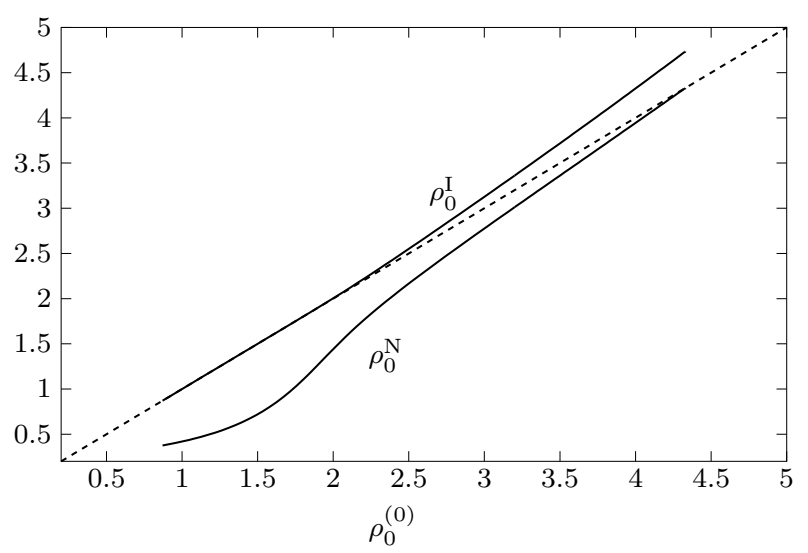

FIG. 7. Number density of the isotropic and nematic phases across the coexistence region for $\sigma=0.7$ and $l_{m}=100$, plotted against the parent density. Notice that in the long rod-dominated region below $\rho_{0}^{(0)} \approx 2$, the density $\rho_{0}^{\mathrm{I}}$ of the isotropic phase is almost identical to that of the parent (dashed line). In the same regime, the nematic density $\rho_{0}^{\mathrm{N}}$ shows a pronounced increase, before crossing over to the more gradual variation typical of systems without fat-tailed length distributions.

The most detailed information about the phase separation is obtained by analysing the density distributions in the coexisting phases. Focussing on the low-density part of the coexistence region that is dominated by the effect of the long rods, we find as expected from Figs. 7 and 8 that the density distribution of the isotropic phase (not shown) is essentially identical to that of the parent, except for a reduction in the already small density of long rods. For the nematic phase, we saw in Fig. 5 that $\rho^{\mathrm{N}}(l)$ has a peak at $l=l_{m}$ at the isotropic cloud point. When we move into the coexistence region by increasing the parent density (Fig. 9), the density distribution acquires a bimodal shape as the second peak gradually moves to smaller values of $l$. Eventually, at the point where the system crosses over to conventional phase behaviour, this second peak merges with the main peak at $l$ of order unity. Before this happens, the nematic phase remains significantly enriched, compared to the parent in some of the longer rods. Since the overall density distribution must be preserved in phase separation (see Eq. (15)), this again makes plausible that the fractional system volume occupied by nematic phase has to be very small in this regime: there simply are not enough long rods to allow formation of a significant amount of a nematic phase that contains a much higher proportion of such long rods.

Finally, we may ask how the properties of the coexisting phases depend on the cutoff $l_{m}$, at some fixed point in the region of the phase diagram where the long rods significantly affect the behaviour (i.e. to the left of the isotropic cloud curve for rod length distributions with- 


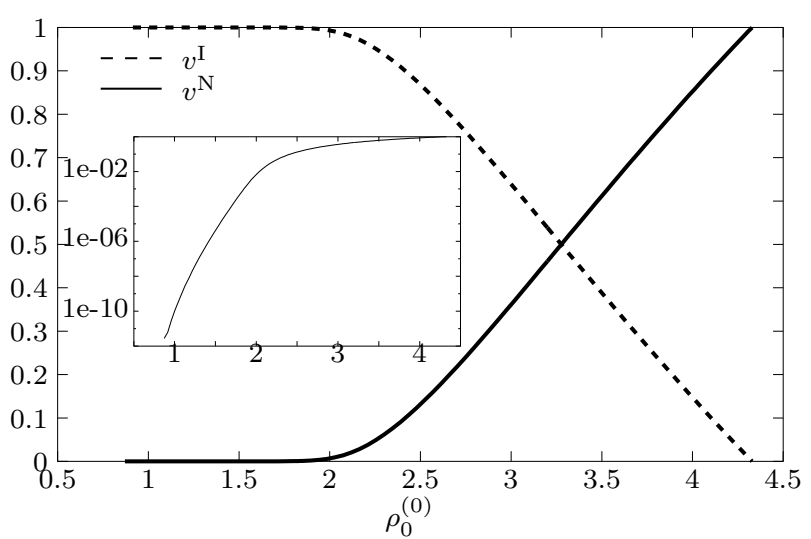

FIG. 8. Fractions of system volume occupied by the isotropic (dashed) and nematic (solid) phases, plotted against parent density across the coexistence region for $\sigma=0.7$ and $l_{m}=100$. On a logarithmic scale (inset) it is clear the in the regime where the phase separation is dominated by the long rods, the fractional volume of the nematic is extremely small.

out fat tails). Naively, one might expect that the cutoff would play an important role not just at the isotropic cloud point, but throughout this long rod-dominated part of the phase diagram. However, this is not so. It turns out, for example, that the peak in $\rho^{\mathrm{N}}(l)$ at large $l$ (but below the cutoff) does not change significantly as $l_{m}$ increases and eventually stabilizes. The same applies to all other properties of the coexisting phases, including the fractional volume $v^{\mathrm{N}}$ of the nematic phase; the latter remains nonzero (though very small) as $l_{m}$ becomes large. Even while the isotropic cloud curve moves towards the limiting curve for infinite cutoff (Eq. (50)), widening the phase coexistence region, the phase separation behaviour in the region beyond the cloud point therefore eventually becomes independent of $l_{m}$.

\section{THEORY FOR ISOTROPIC CLOUD POINT}

Having discussed the phase behaviour of systems with a log-normal parent distribution with a finite length cutoff $l_{m}$, as obtained from our numerical calculations, we now turn to the limit of large $l_{m}$ where the onset of nematic ordering, i.e. the properties of the isotropic cloud and nematic shadow phases, are amenable to theoretical treatment. Even though any physical system will necessarily have a finite largest rod length and therefore a finite $l_{m}$, the limiting case $l_{m} \rightarrow \infty$ is useful in understanding some of the physics that we have described in the previous sections. In particular, we can extract a large amount of information about the characteristics of the coexisting phases, such as the scaling of all the densities of the coexisting phases with $l_{m}$, which can be compared directly with our numerical results. The cloud
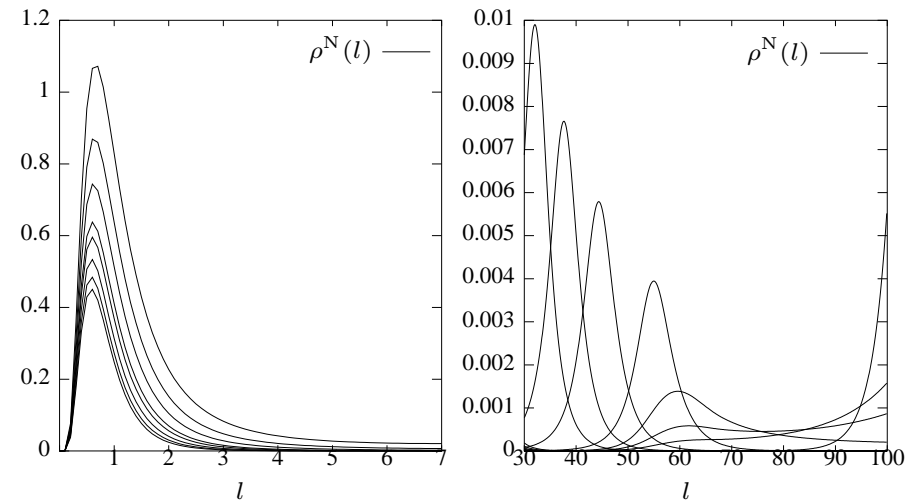

FIG. 9. Plot of the density distribution of the nematic phase for short (left) and long (right) rods, for a range of parent densities across the coexistence region at $\sigma=0.28$ and $l_{m}=100$. With increasing parent density, i.e. when moving away from the isotropic cloud point, the first (left) peak of the distribution grows and approaches the shape of the parent. The second peak at $l_{m}$, on the other hand, first decreases in height and then gives way to a maximum at $l<l_{m}$ which moves towards smaller $l$. The crossover to the region in the phase diagram where long rods no longer significantly affect the phase behaviour takes place when this maximum merges with the main maximum on the left.

and shadow curves can be deduced explicitly in the limit $l_{m} \rightarrow \infty$ and actually coincide. The moment density $\rho_{2}^{N}$ of the nematic shadow phase vanishes in the limit. Since $\rho_{2}^{\mathrm{N}}$ specifies the degree of orientational ordering, one may doubt whether this solution can justifiably be referred to as a nematic phase. However, recall from Eq. (6) that $\rho_{2}^{\mathrm{N}}$ is just $\rho_{0}^{\mathrm{N}}\langle S(l) l\rangle$, where the average is taken over the normalized length distribution in the nematic, $P^{\mathrm{N}}(l)=\rho^{\mathrm{N}}(l) / \rho_{0}^{\mathrm{N}}$. A small value of $\rho_{2}^{\mathrm{N}}$ then implies that $S(l)$, the orientational order parameter for rods of length $l$ defined in Eq. (7), must be close to zero wherever $P^{\mathrm{N}}(l)$ is non-vanishing. In regions where $P^{\mathrm{N}}(l)$ has little weight, on the other hand, the value of $S(l)$ can be close to one. This is exactly what happens in the nematic shadow phase in the limit $l_{m} \rightarrow \infty$. For $l$ of order unity $P^{\mathrm{N}}(l)$ has nonzero weight as can be seen from the plot of $\rho^{\mathrm{N}}(l)=\rho_{0}^{\mathrm{N}} P^{\mathrm{N}}(l)$ in Fig. 5. Correspondingly, $S(l) \rightarrow 0$ in this regime of rod lengths, implying that the short rods are orientationally disordered. For the longest rods with $l$ of order $l_{m}$, on the other hand, $P^{\mathrm{N}}(l)$ has negligible weight for $l_{m} \rightarrow \infty$, and we will find that these rods are strongly ordered, with $S(l) \rightarrow 1$. The contributions to $\rho_{2}^{\mathrm{N}}$ from both regimes vanish in the limit, although nontrivially they are of the same order; the fact that the length distribution has much more weight on short rods is compensated by the smallness of $S(l)$ there.

In the next sections we will establish, by a process of elimination, the overall limiting behaviour of the relevant quantities as $l_{m}$ becomes large. Once we have derived the system of equations to be solved for finite $l_{m}$, we 
will decompose all integrals over $l$ into two parts, one for lengths of order unity, $l=0 \ldots \tilde{l}$ and one for the large lengths $l=\tilde{l} \ldots l_{m}$. Here $\tilde{l}$ is chosen such that $l_{m} \gg \tilde{l} \gg 1$; formally, we first take $l_{m} \rightarrow \infty$ and then $\tilde{l} \rightarrow \infty$. The decomposition into the two parts allows us to simplify all integrals, making use of the fact that the short rods are orientationally disordered while the long ones are strongly ordered. From this we eventually derive the solution for the isotropic cloud point and the scaling of all quantities with $l_{m}$.

\section{A. Asymptotic behaviour of $\beta$ and $\rho_{2}^{\mathrm{N}}$}

Let us start our analysis with a discussion of the behaviour, in the limit $l_{m} \rightarrow \infty$, of the key quantity $\beta$; from Eq. (18) it follows that its value has a strong effect on the properties of the nematic shadow phase. Explicitly, we have from Eq. (18) that the moments of the nematic shadow phase are

$$
\begin{aligned}
& \rho_{0}^{\mathrm{N}}=\rho_{0} \int d l \widetilde{d \theta} P^{(0)}(l) e^{\beta l} e^{l f(\theta)} \\
& \rho_{1}^{\mathrm{N}}=\rho_{0} \int d l \widetilde{d \theta} P^{(0)}(l) l e^{\beta l} e^{l f(\theta)} \\
& \rho_{2}^{\mathrm{N}}=\rho_{0} \int d l \widetilde{d \theta} P^{(0)}(l) l e^{\beta l} \mathcal{P}_{2}(\cos \theta) e^{l f(\theta)}
\end{aligned}
$$

where we have defined $f(\theta)$ as

$$
f(\theta)=c_{2} \rho_{2}^{\mathrm{N}}\left(\mathcal{P}_{2}-1\right)
$$

and have used the fact that the isotropic phase coincides with the parent distribution $\rho^{(0)}(l)$ at the isotropic cloud point; $\rho_{0}=\int d l \rho^{(0)}(l)$ is the parent density (we drop the superscript on $\rho_{0}^{(0)}$ for brevity). An important constraint on the dependence of $\beta$ on $l_{m}$ is that all the above moment densities must remain finite as $l_{m} \rightarrow \infty$. This follows from the equality of the osmotic pressure (13), which gives

$$
\rho_{0}+\frac{1}{2} c_{1} \rho_{0}^{2}=\rho_{0}^{\mathrm{N}}+\frac{1}{2} c_{1}\left(\rho_{1}^{\mathrm{N}}\right)^{2}-\frac{1}{2} c_{2}\left(\rho_{2}^{\mathrm{N}}\right)^{2}
$$

On the 1.h.s. we have used again that the isotropic cloud phase coincides with the parent, giving $\rho_{0}^{\mathrm{I}}=\rho_{0}$ and also $\rho_{1}^{\mathrm{I}}=\rho_{0}^{\mathrm{I}}=\rho_{0}$ since the average rod length in the parent is unity. All terms on the l.h.s. of Eq. (13) are finite. The r.h.s. is $\geq \rho_{0}^{\mathrm{N}}+\left(c_{1}-c_{2}\right)\left(\rho_{1}^{\mathrm{N}}\right)^{2} / 2$ since $\rho_{2}^{\mathrm{N}} \leq \rho_{1}^{\mathrm{N}}$ from Eqs. $(23,24)$. Using $c_{1}>c_{2}$ it follows that both terms are positive, so that $\rho_{0}^{\mathrm{N}}$ and $\rho_{1}^{\mathrm{N}}$, and therefore also $\rho_{2}^{\mathrm{N}}$, must remain finite as claimed. As in Sec. II B, we can now deduce that a length cutoff $l_{m}$ must be imposed on all integrals over $l$ whenever the parent distribution is less than exponentially decaying at large $l$. This follows because $\beta$ should be positive in order to have a greater rod volume fraction in the nematic phase than in the isotropic, i.e. $\rho_{1}^{\mathrm{N}} \geq \rho_{1}^{\mathrm{I}}$. Without a cutoff on $l$, such a positive value of $\beta$ in Eqs. $(22,23)$ would lead to divergent integrals for $\rho_{0}^{\mathrm{N}}$ and $\rho_{1}^{\mathrm{N}}$, violating the constraint that these moment densities must be finite. (The angular integrals cannot counteract the effect of the exponential factor $\exp (\beta l)$, since they vary at most as power laws with $l$.) The cutoff $l_{m}$ will be assumed large and later taken to infinity to study the limiting behaviour for large cutoffs. Initially, we develop the theory for general parent distributions with fat tails, but then eventually specialize to the log-normal case in order to compare with our numerical results for finite cutoffs ${ }^{36}$.

We are now in a position to narrow down the limiting behaviour of $\beta$ as $l_{m} \rightarrow \infty$. With $\beta$ being positive, there are three possibilities: $\beta \rightarrow \infty, \beta \rightarrow$ const. and $\beta \rightarrow 0$. The first case can be excluded from Eq. (19), which using $\rho_{1}^{\mathrm{I}}=\rho_{0}$ becomes

$$
\beta=-c_{1}\left(\rho_{1}^{\mathrm{N}}-\rho_{0}\right)+c_{2} \rho_{2}^{\mathrm{N}}
$$

We have already established that all terms on the r.h.s. of this equation remain finite, so that $\beta$ cannot diverge. In the second case, $\beta \rightarrow$ const. $>0$, the integral in Eq. (22) for $\rho_{0}^{\mathrm{N}}$ will diverge as $l_{m} \rightarrow \infty$, giving $\rho_{0}^{\mathrm{N}} / \rho_{0} \rightarrow \infty$ and therefore $\rho_{0} \rightarrow 0$ to keep $\rho_{0}^{\mathrm{N}}$ finite. But then the 1.h.s. of the osmotic pressure equality $(26)$ becomes $\simeq \rho_{0}$, while the r.h.s. is $\geq \rho_{0}^{\mathrm{N}}+\left(c_{1}-c_{2}\right)\left(\rho_{1}^{\mathrm{N}}\right)^{2} / 2 \geq \rho_{0}^{\mathrm{N}}$ as explained above; the pressure equality can therefore not be satisfied since $\rho_{0}^{\mathrm{N}} \gg \rho_{0}$. We therefore conclude that the only possible limit behaviour is $\beta \rightarrow 0$ for $l_{m} \rightarrow \infty$. This means that in the "short rods" region, i.e. for fixed values of the rod length $l$ that do not grow with $l_{m}, \beta l$ is a small parameter in which we can expand. The limiting behaviour of the product $\beta l_{m}$ is more difficult to determine, although it is plausible that if this quantity is not large enough then the supposed nematic shadow phase will behave as if $\beta=0$ exactly and thus become fully identical to the isotropic cloud phase in the limit, implying that no genuine phase coexistence has been found. We therefore assume in the following that $\beta l_{m} \rightarrow \infty$ for $l_{m} \rightarrow \infty$, and will find support for this in the numerical results presented in Sec. IV F.

Let us now turn to the behaviour of $\rho_{2}^{N}$ for $l_{m} \rightarrow \infty$. Having shown that it cannot diverge, the two possibilities are that $\rho_{2}^{\mathrm{N}} \rightarrow$ const. $>0$ or $\rho_{2}^{\mathrm{N}} \rightarrow 0$. In the first case we show in App. A that the problem can be drastically reduced to a single equation in $\rho_{2}^{\mathrm{N}}$. The only solution of this equation is $\rho_{2}^{\mathrm{N}}=0$, in contradicition with the assumed finite limit value. We therefore conclude that both $\beta$ and $\rho_{2}^{\mathrm{N}}$ vanish in the limit of infinite cutoff. As explained above, we will see that the nematic shadow phase remains a bona fide nematic in spite of the fact that $\rho_{2}^{\mathrm{N}} \rightarrow 0$ : the longest rods exhibit strong nematic order but their contribution to $\rho_{2}^{\mathrm{N}}$ vanishes in the limit. As pointed out earlier, the fact that $\rho_{2}^{\mathrm{N}} \rightarrow 0$ also demonstrates that the short rods in the nematic shadow phase are orientationally disordered. 


\section{B. Setup of the equations}

We now proceed to simplify the equation for the isotropic cloud and nematic shadow phases, using that $\beta$ and $\rho_{2}^{\mathrm{N}}$ both converge to zero for large $l_{m}$ while $\beta l_{m}$ diverges. The equations to be solved are $(22,23,24)$ for the moments of the nematic shadow phase, the osmotic pressure equality (26) and the definition (27) of $\beta$. These five equations determine the five unknowns $\rho_{0}, \rho_{0}^{\mathrm{N}}, \rho_{1}^{\mathrm{N}}$, $\rho_{2}^{\mathrm{N}}$ and $\beta$ as a function of the cutoff $l_{m}$. Equivalently, we can use Eqs. $(22,23)$ to eliminate $\rho_{0}^{\mathrm{N}}$ and $\rho_{1}^{\mathrm{N}}$, solving the three remaining equations for $\rho_{0}, \rho_{2}^{\mathrm{N}}$ and $\beta$.

We now proceed to implement our scheme of breaking the integrals over $l$ into two intervals, namely $l=0 \ldots l$ and $l=\tilde{l} \ldots l_{m}$, where $1 \ll \tilde{l} \ll l_{m}$. Calling $I_{0}$ the contribution of the long rods to $\rho_{0}^{\mathrm{N}}$, Eq. (22) becomes in this way

$$
\rho_{0}^{\mathrm{N}}=\rho_{0} \int_{0}^{\tilde{l}} d l P^{(0)}(l) e^{\beta l} \int \widetilde{d \theta} e^{l f(\theta)}+I_{0}
$$

where

$$
I_{0}=\rho_{0} \int_{\tilde{l}}^{l_{m}} d l P^{(0)}(l) e^{\beta l} \int \widetilde{d \theta} e^{l f(\theta)}
$$

In the integral over the short rods we can expand in the small parameters $\beta l$ and $\rho_{2}^{\mathrm{N}} l$. Since from Eq. $(25) f(\theta) l$ is proportional to $\rho_{2}^{\mathrm{N}} l$, an expansion to linear order gives

$$
\rho_{0}^{\mathrm{N}}=\rho_{0} \int_{0}^{\tilde{l}} d l P^{(0)}(l) \int \widetilde{d \theta}\{1+\beta l+f(\theta) l\}+I_{0}
$$

Having isolated the contribution from the long rods, we can now extend the integration to $\infty$ in the convergent l-integral in the first term; the error from this approximation turns out to be negligible compared to the cutoffdependent terms that we focus on. This gives, using that the average parental rod length is unity,

$$
\rho_{0}^{\mathrm{N}}=\rho_{0}+\left(\beta-c_{2} \rho_{2}^{\mathrm{N}}\right) \rho_{0}+I_{0}
$$

The same procedure can be applied to Eqs. $(23,24)$ and yields

$$
\begin{aligned}
& \rho_{1}^{\mathrm{N}}=\rho_{0}+\left(\beta-c_{2} \rho_{2}^{\mathrm{N}}\right)\left\langle l^{2}\right\rangle \rho_{0}+I_{1} \\
& \rho_{2}^{\mathrm{N}}=\frac{c_{2}}{5} \rho_{0}\left\langle l^{2}\right\rangle \rho_{2}^{\mathrm{N}}+I_{2}
\end{aligned}
$$

where the long rod parts are

$$
\begin{aligned}
& I_{1}=\rho_{0} \int_{\tilde{l}}^{l_{m}} d l l P^{(0)}(l) e^{\beta l} e^{l f(\theta)} \\
& I_{2}=\rho_{0} \int_{\tilde{l}}^{l_{m}} d l l P^{(0)}(l) e^{\beta l} \mathcal{P}_{2} e^{l f(\theta)}
\end{aligned}
$$

and the averages $\left\langle l^{n}\right\rangle$ are taken over the normalized parent distribution

$$
\left\langle l^{n}\right\rangle=\int d l l^{n} P^{(0)}(l)
$$

The long rod contributions $I_{0}, I_{1}, I_{2}$ remain undetermined so far. They must certainly be finite for $l_{m} \rightarrow \infty$, because the moments are, but in fact will turn out to converge to zero. For $I_{2}$ this is easy to see from Eq. (31), which implies that $I_{2} \leq \rho_{2}^{\mathrm{N}}$. We can further exclude the possibility that $I_{2} \ll \rho_{2}^{\mathrm{N}}$, which would imply from Eq. (31) that

$$
\left\langle l^{2}\right\rangle \rho_{0}=4
$$

It is easy to show that this is just the spinodal condition for the isotropic phase in the $\mathcal{P}_{2}$ Onsager model, while the onset of isotropic-nematic phase coexistence must happen at a density below the spinodal instability. Thus, $I_{2}$ must be of the same order as $\rho_{2}^{\mathrm{N}}$, which means that short and long rods make comparable contributions to $\rho_{2}^{\mathrm{N}}$ as anticipated above.

To evaluate the integrals for $I_{0}, I_{1}$ and $I_{2}$ more quantitatively, we note that they all contain the exponentially diverging factor $\exp (\beta l)$ which dominates the behaviour of the integrands since $\beta l_{m} \rightarrow \infty$. To leading order we can thus approximate the integrands $P^{(0)}(l) w(l) e^{\beta l}$, where $w(l)$ is one of the weight functions in the above Eqs. $(28,32,33)$, by $P^{(0)}\left(l_{m}\right) w\left(l_{m}\right) e^{\beta l}$ and integrate. For the log-normal parent distribution we show in App. B that this approximation indeed becomes exact for $l_{m} \rightarrow$ $\infty$. For more quickly decaying distributions, for example $P^{(0)}(l) \sim \exp \left(-l^{\alpha}\right)$ with $0<\alpha<1$, a slightly better approximation is needed (see App. B), but the integrals remain dominated by their values at $l=l_{m}$.

It follows from the above arguments that $I_{0}$ is smaller than $I_{1}$ by a factor of $1 / l_{m}$ for large $l_{m}$, and can therefore be neglected to leading order. Likewise, the ratio of $I_{2}$ and $I_{1}$ becomes simply

$$
s=\frac{I_{2}}{I_{1}}=\frac{\int \widetilde{d \theta} \mathcal{P}_{2} e^{l_{m} f(\theta)}}{\int \widetilde{d \theta} e^{l_{m} f(\theta)}}
$$

which is in fact identical to $S\left(l_{m}\right)$, the orientational order parameter for the longest rods, and therefore lies between 0 and 1 . Using the definition of $s$, Eq. (31) can be written as

$$
\rho_{2}^{\mathrm{N}}=\frac{c_{2}}{5} \rho_{0}\left\langle l^{2}\right\rangle \rho_{2}^{\mathrm{N}}+s I_{1}
$$

which will be useful shortly.

So far we have only dealt with the equations for the moment densities $\rho_{0}^{\mathrm{N}}, \rho_{1}^{\mathrm{N}}$ and $\rho_{2}^{\mathrm{N}}$ of the nematic phase. We now add Eq. (27) for $\beta$; inserting Eq. (30) and bringing all terms in $\beta$ to the left gives

$$
\beta\left(1+c_{1}\left\langle l^{2}\right\rangle \rho_{0}\right)=c_{2} \rho_{2}^{\mathrm{N}}\left(1+c_{1}\left\langle l^{2}\right\rangle \rho_{0}\right)-c_{1} I_{1}
$$

This implies $\beta \leq c_{2} \rho_{2}^{\mathrm{N}}$, and since $\beta l_{m} \rightarrow \infty$ for $l_{m} \rightarrow \infty$ we also have $\rho_{2}^{\mathrm{N}} \bar{l}_{m} \rightarrow \infty$. Recalling $f(\theta)=c_{2} \rho_{2}^{\mathrm{N}}\left(\mathcal{P}_{2}-1\right)$, we therefore see from (34) that the long rods have strong 
orientational order. More quantitatively, by expanding $\mathcal{P}_{2}-1 \simeq-\frac{3}{2} \theta^{2}$ and performing the resulting Gaussian integrals, we find that

$$
s=1-\frac{1}{c_{2} \rho_{2}^{\mathrm{N}} l_{m}}
$$

to first order in $1 /\left(\rho_{2}^{\mathrm{N}} l_{m}\right)$. To leading order, one has $s=1$ and Eq. (35) simplifies to

$$
I_{1}=C \rho_{2}^{\mathrm{N}}
$$

with

$$
C=1-\frac{c_{2}}{5} \rho_{0}\left\langle l^{2}\right\rangle
$$

Using this in Eq. (36) we get:

$$
\beta=\gamma \rho_{2}^{\mathrm{N}}
$$

where

$$
\gamma=\frac{(6 / 5) c_{1} c_{2} \rho_{0}\left\langle l^{2}\right\rangle+c_{2}-c_{1}}{1+c_{1} \rho_{0}\left\langle l^{2}\right\rangle}
$$

For our set of variables $\rho_{0}, \rho_{2}^{\mathrm{N}}$ and $\beta$ we now have a set of three equations, consisting of the simplified Eqs. (38) and (40) and the osmotic pressure equality (26). Before turning to the latter, we note that Eq. (41) implies that $\beta$ is of the same order as $\rho_{2}^{N}$ for $l_{m} \rightarrow \infty$ unless $\gamma \rightarrow 0$ in the same limit. We will find below that the second alternative holds, and that $\beta$ is actually proportional to $\left(\rho_{2}^{\mathrm{N}}\right)^{2}$.

Consider now the pressure equality, given by Eq. (26) in its still exact form. We would like to apply to this the same procedure of splitting the $l$-integrals into contributions from short and long rods, and then expanding in $\beta l$ and $\rho_{2}^{\mathrm{N}} l$ in the short rods part. It turns out, however, that the expansion in the short rods part needs to be carried to third order in order to give an equation which is independent of the ones we have already derived. We therefore first rearrange the pressure equality into a form where the linear and quadratic terms in the expansion cancel automatically.

On the l.h.s. of Eq. (26), we write $\rho_{0}=\int d l \rho^{(0)}(l)$; in the quadratic term we similarly replace one of the factors using $\rho_{0}=\rho_{1}=\int d l \rho^{(0)}(l) l$. The r.h.s. can be rewritten in a similar way, using the expressions $(22,23,24)$ for the moments of the nematic phase. Including an overall factor of 2, the osmotic pressure equality then takes the form

$$
\begin{aligned}
0= & \int d l \widetilde{d \theta} \rho^{(0)}(l)\left\{e^{\beta l+f(\theta) l}\left[2+c_{1} \rho_{1}^{\mathrm{N}} l-c_{2} \rho_{2}^{\mathrm{N}} l \mathcal{P}_{2}\right]\right. \\
& \left.-2-c_{1} \rho_{0} l\right\}
\end{aligned}
$$

and the definitions (25) and (27) can be used to write the last two terms in the square bracket as

$$
c_{1} \rho_{1}^{\mathrm{N}} l-c_{2} \rho_{2}^{\mathrm{N}} l \mathcal{P}_{2}=\left[-f(\theta)-\beta+c_{1} \rho_{0}\right] l
$$

In the same way, the definition (19) of $\beta$ can be written in integral form as

$$
\beta=\int d l \widetilde{d \theta} \rho^{(0)}(l) c_{1} l\left[1-e^{\beta l+f(\theta) l}\right]+c_{2} \rho_{2}^{\mathrm{N}}
$$

Multiplying by $\rho_{0}=\int d l \rho^{(0)}(l) l$ and bringing all terms to the r.h.s. gives

$0=\int d l \widetilde{d \theta} \rho^{(0)}(l)\left\{-\beta l+c_{1} \rho_{0} l\left[1-e^{\beta l+f(\theta) l}\right]+c_{2} \rho_{2}^{\mathrm{N}} l\right\}$

If we now sum Eq. (42) and Eq. (44), and make use of Eq. (43) and the fact that $c_{2} \rho_{2}^{\mathrm{N}}=-\int \widetilde{d \theta} f(\theta)$, we obtain after a few rearrangements

$$
\begin{aligned}
0= & \int d l \widetilde{d \theta} \rho^{(0)}(l)\left\{2\left[e^{\beta l+f(\theta) l}-1\right]\right. \\
& \left.-[\beta+f(\theta)] l\left[e^{\beta l+f(\theta) l}+1\right]\right\}
\end{aligned}
$$

Eq. (45) is the desired form of the osmotic pressure equality, which is still exact. We can now proceed as above and split the $l$-integral into the contributions from short and long rods. In the short rods part, we Taylor expand the integrand in the small variable $[\beta+f(\theta)] l$; as anticipated above, the first nonzero term in this expansion is of third order. To leading order, Eq. (45) then becomes

$$
0=-\int_{0}^{\tilde{l}} d l \widetilde{d \theta} \rho^{(0)}(l) \frac{l^{3}}{6}[\beta+f(\theta)]^{3}+I_{3}
$$

where $I_{3}$ represents the long rods part of the integral in Eq. (45). In the first term we can again take the upper integration limit to $\infty$ and obtain

$$
-\frac{\left\langle l^{3}\right\rangle}{6} \rho_{0} \int \widetilde{d \theta}[\beta+f(\theta)]^{3}=-I_{3}
$$

where as above the average $\left\langle l^{3}\right\rangle$ is over the normalized parent distribution $P^{(0)}(l)$.

Our final task in simplifying the pressure equality is to find $I_{3}$. Because $\beta l_{m}$ diverges (and we have restricted the integration range to the long rods) the exponential factors in Eq. (45) dominate the integral. Of the terms involving these factors, the first one is also negligible compared to the second one, $-[\beta+f(\theta)] l e^{\beta l+f(\theta) l}$, since it is missing the large factor $[\beta+f(\theta)] l$. Comparing with Eqs. $(32,33)$, we thus have that

$$
I_{3}=-\left[\beta I_{1}+c_{2} \rho_{2}^{\mathrm{N}}\left(I_{2}-I_{1}\right)\right]=-\left[\beta+c_{2} \rho_{2}^{\mathrm{N}}(s-1)\right] I_{1}
$$

for large $l_{m}$. Eq. (37) further implies that $c_{2} \rho_{2}^{\mathrm{N}}(s-1)=$ $-1 / l_{m}$ to leading order, which is negligible compared to $\beta$ since $\beta l_{m} \rightarrow \infty$. Altogether we have the simple result $I_{3}=-\beta I_{1}$, and the transformed pressure equality (45) becomes in its final form

$$
-\frac{\left\langle l^{3}\right\rangle}{6} \rho_{0} \int \widetilde{d \theta}\left[\beta+c_{2} \rho_{2}^{\mathrm{N}}\left(\mathcal{P}_{2}-1\right)\right]^{3}=\beta I_{1}
$$




\section{The isotropic cloud point}

In the previous subsection, we used the large cutofflimit to simplify the three basic equations for the three variables $\rho_{0}, \rho_{2}^{\mathrm{N}}$ and $\beta$, obtaining Eq. (38) for $\rho_{2}$, Eq. (40) for $\beta$ and the transformed pressure equality (48) for $\rho_{0}$. While in principle these equations need to be solved simultaneously, the limiting value of the isotropic cloud point is easy to extract directly, as follows. Using Eqs. $(38,40)$ to eliminate $\beta$ and $I_{1}$, and dividing by $\left(\rho_{2}^{\mathrm{N}}\right)^{2}$ the pressure equality (48) becomes

$$
-\frac{\left\langle l^{3}\right\rangle}{6} \rho_{0} \rho_{2}^{\mathrm{N}} \int \widetilde{d \theta}\left[\gamma+c_{2}\left(\mathcal{P}_{2}-1\right)\right]^{3}=C \gamma
$$

The 1.h.s. of this equation tends to zero for $l_{m} \rightarrow \infty$ since $\rho_{2}^{\mathrm{N}}$ does; in the same limit we must therefore have $C \gamma=0$. Thus either $C=0$ or $\gamma=0$; but the first case we have already excluded in our discussion after Eq. (31) as leading to the spinodal instability rather than the cloud point. Thus $\gamma=0$, and inserting $c_{1}=2$ and $c_{2}=5 / 4$ into Eq. (41) gives

$$
\left\langle l^{2}\right\rangle \rho_{0}=\frac{1}{4}
$$

as our final result for the parent density $\rho_{0}$ at the isotropic cloud point, in the large cutoff limit $l_{m} \rightarrow \infty$. From the definition (21) of the polydispersity $\sigma$, and bearing in mind that we took the average rod length in the parent to be unity, we have $\left\langle l^{2}\right\rangle=\sigma^{2}+1$ so that the cloud point condition can also be written as

$$
\rho_{0}=\frac{1}{4} \frac{1}{\sigma^{2}+1}
$$

The result $\gamma \rightarrow 0$ for $l_{m} \rightarrow \infty$ implies, from Eq. (40), that $\beta \ll \rho_{2}^{\mathrm{N}}$ for large $l_{m}$. This relation helps to understand the appearance of the minus sign on the 1.h.s. of the transformed pressure equality (48). Call $g(\theta)=$ $\beta+c_{2} \rho_{2}^{\mathrm{N}}\left(\mathcal{P}_{2}-1\right)$ the function appearing in the integrand. Then $\beta=g(0)>0$ while for other $\theta$ the value of $g(\theta)$ is lower. In fact, if $\beta \ll \rho_{2}^{\mathrm{N}}$, then $g(\theta)$ is negative except in a very small region around $\theta=0$ (and of course $\theta=\pi$ ). These negative values of $g(\theta)$ will dominate the integral on the l.h.s. of Eq. (48) and cause it to be negative, making the 1.h.s. positive overall. Interestingly, the situation on the r.h.s. is the reverse. As discussed above, for large $l_{m}$ we can write

$$
\begin{aligned}
-I_{3} & =\int d l \widetilde{d \theta} \rho^{(0)}(l)[\beta+f(\theta)] l e^{\beta l+f(\theta) l} \\
& =\int d l \widetilde{d \theta} \rho^{(0)}(l) g(\theta) l e^{g(\theta) l}
\end{aligned}
$$

Since the integral here is over the long rods only, and in addition $g(0) l_{m}=\beta l_{m} \rightarrow \infty$ for $l_{m} \rightarrow \infty$, the exponential factor ensures that the integral is dominated entirely by the small region of angles around $\theta=0$ (and $\theta=\pi$ ) where $g(\theta)$ is positive.
Having seen that $\beta \ll \rho_{2}^{\mathrm{N}}$ for large $l_{m}$, we can now show that in fact $\beta \sim\left(\rho_{2}^{N}\right)^{2}$. On the 1.h.s. of Eq. (48) we can neglect the first term in square brackets and integrate the remainder explicitly to obtain $(54 / 35)\left(\left\langle l^{3}\right\rangle / 6\right) \rho_{0} c_{2}^{3}\left(\rho_{2}^{\mathrm{N}}\right)^{3}$. On the r.h.s. nothing changes and we can use Eq. (38) to eliminate $I_{1}$. Dividing by $\rho_{2}^{\mathrm{N}}$, Eq. (48) thus becomes

$$
\beta=\kappa\left(\rho_{2}^{\mathrm{N}}\right)^{2}, \quad \kappa=\frac{9}{35} \frac{\left\langle l^{3}\right\rangle \rho_{0} c_{2}^{3}}{C}
$$

showing that indeed $\beta \propto\left(\rho_{2}^{N}\right)^{2}$.

\section{Scaling of $\beta$ and $\rho_{2}^{N}$}

So far we have only used our simplified equations for the isotropic cloud point to deduce the value of the cloud point density for $l_{m} \rightarrow \infty$. The equations were obtained by expanding the short rod integrals in the equations for $\rho_{2}^{\mathrm{N}}$ and $\beta$ to linear order in these two variables; in the osmotic pressure equality we needed to expand to third order because lower terms cancel out automatically. From the expanded pressure equality we deduced that the coefficient $\gamma$ relating $\beta$ and $\rho_{2}^{\mathrm{N}}$ to linear order has to vanish in the limit $l_{m} \rightarrow \infty$, and that in fact $\beta$ must be proportional to $\left(\rho_{2}^{\mathrm{N}}\right)^{2}$ to leading order; the condition $\gamma=$ 0 gave us the limiting value of the cloud point density for infinite cutoff. These results all apply generally for parent rod length distributions with fat (less than exponentially decaying) tails.

We now proceed to obtain from our equations additional information regarding the variation of the key quantities with the cutoff $l_{m}$, in the regime of large $l_{m}$. These quantities are $\beta, \rho_{2}^{\mathrm{N}}$ and the moment densities of the isotropic cloud and nematic shadow phases. To do so, we have to explicitly calculate the long rod contribution

$$
I_{1}=\rho_{0} \int_{\tilde{l}}^{l_{m}} d l P^{(0)}(l) l e^{\beta l} \int \widetilde{d \theta} e^{l f(\theta)}
$$

to $\rho_{1}^{\mathrm{N}}$, which so far we had left unevaluated. Anticipating that the integral is dominated by rod lengths of order $l_{m}$, for which $\rho_{2}^{\mathrm{N}} l$ is large since $\rho_{2}^{\mathrm{N}} l_{m} \rightarrow \infty$ as $l_{m} \rightarrow \infty$, we can evaluate the angular integral by expanding around $\theta=0$ (see before Eq. (37)) to get

$$
I_{1}=\rho_{0} \int_{\tilde{l}}^{l_{m}} d l P^{(0)}(l) \frac{e^{\beta l}}{3 c_{2} \rho_{2}^{\mathrm{N}}}
$$

It will be useful to remove the overall factors and define the quantity

$$
\tilde{I}_{1}=\frac{3 c_{2} \rho_{2}^{\mathrm{N}}}{\rho_{0}} I_{1}=\int_{\tilde{l}}^{l_{m}} d l P^{(0)}(l) e^{\beta l}
$$

which only depends on $\beta$ and $l_{m}$. From Eq. (38) we then have $\tilde{I}_{1}=\left(3 c_{2} C / \rho_{0}\right)\left(\rho_{2}^{\mathrm{N}}\right)^{2}$, and using Eq. (51) to eliminate $\rho_{2}^{\mathrm{N}}$ in favour of $\beta$ gives 


$$
\frac{\tilde{I}_{1}}{\beta}=A, \quad A=\frac{3 c_{2} C}{\rho_{0} \kappa}
$$

All factors in the definition of $A$, and therefore $A$ itself, tend to nonzero constants as $l_{m} \rightarrow \infty$; the above equation therefore implicitly determines the asymptotic dependence of $\beta$ on $l_{m}$.

To evaluate $\tilde{I}_{1}$, one notes (see App. B) that for fattailed parent distributions such as the log-normal the exponentially increasing factor $\exp (\beta l)$ dominates the behaviour of the integrand. The integral is therefore dominated by the region $l \approx l_{m}$ and one can replace $l=l_{m}$ in the non-exponential factors of the integrand, yielding

$$
\tilde{I}_{1}=P^{(0)}\left(l_{m}\right) \frac{e^{\beta l_{m}}}{\beta}
$$

for large $l_{m}$. Eq. (54) then gives

$$
\frac{e^{\beta l_{m}}}{\left(\beta l_{m}\right)^{2}}=\frac{A}{l_{m}^{2} P^{(0)}\left(l_{m}\right)}
$$

The function $e^{x} / x^{2}$ on the lhs of Eq. (56) can be inverted for large $\operatorname{argument} x$ and yields asymptotically just a logarithm (see App. B), so that

$$
\beta=\frac{1}{l_{m}} \ln \left(\frac{A}{l_{m}^{2} P^{(0)}\left(l_{m}\right)}\right)
$$

Now for a log-normal parent distribution $\ln P^{(0)}(l)=$ $-\ln ^{2} l_{m} /\left(2 w^{2}\right)$ plus terms that are negligible by comparison for large $l_{m}$, giving for the leading asymptotic dependence of $\beta$ on $l_{m}$

$$
\beta=\frac{\ln ^{2} l_{m}}{2 l_{m} w^{2}}
$$

For a more general parent distribution with a fat tail, i.e. $P^{(0)}(l)=e^{-h(l)}$ with $h(l)$ a function that diverges with $l$ less than linearly, the approximation (55) is not quite correct but can be refined (see App. B) to

$$
\tilde{I}_{1}=\frac{e^{\beta l_{m}-h\left(l_{m}\right)}}{\beta-h^{\prime}\left(l_{m}\right)}
$$

Inserting into Eq. (54), one can again solve for $\beta$ and obtains asymptotically

$$
\beta=\frac{h\left(l_{m}\right)}{l_{m}}
$$

which generalizes Eq. (58). For $h(l) \sim l^{\alpha}$ with $0<\alpha<1$, which corresponds to a parent distribution decaying more quickly than a log-normal but still less than exponentially, one gets $\beta \sim l_{m}^{\alpha-1}$. Using that $\beta \propto\left(\rho_{2}^{\mathrm{N}}\right)^{2}$ for large $l_{m}$, the above results also imply that $\rho_{2}^{\mathrm{N}} \sim\left[h\left(l_{m}\right) / l_{m}\right]^{1 / 2}$, with $\rho_{2}^{\mathrm{N}} \propto l_{m}^{-1 / 2} \ln l_{m}$ for the log-normal case. Notice that the results for $h(l) \sim l^{\alpha}$ extrapolate sensibly towards the case of a parent distribution without a fat tail, i.e. $\alpha=1$.
Then neither $\beta$ nor $\rho_{2}^{\mathrm{N}}$ have any reason to tend to zero for $l_{m} \rightarrow \infty$, and this is consistent with the limit $\alpha \rightarrow 1$ of the scalings above. As explained in Sec. III C, the scaling of $\rho_{2}^{\mathrm{N}}$ also determines directly the length-dependence of orientational order in the nematic phase: only rods with $l$ around the "ordering length" $1 / \rho_{2}^{\mathrm{N}}$ and greater show significant ordering. For the log-normal case, the ordering length is $1 / \rho_{2}^{\mathrm{N}} \sim l_{m}^{1 / 2} / \ln l_{m}$ and $\gg 1$ as expected, but simultaneously $\ll l_{m}$. This makes precise our earlier statements that orientational order is confined to the longer rods in the nematic phase; ordering appears for rod lengths far above unity, but still well below the cutoff length $l_{m}$.

\section{E. Scaling of densities}

Finally, we study the scaling with $l_{m}$ of the isotropic cloud point density $\rho_{0}$ and the number density $\rho_{0}^{\mathrm{N}}$ and rescaled volume fraction $\rho_{1}^{\mathrm{N}}$ of the nematic shadow phase. From Eqs. $(29,30)$, with $\beta \ll \rho_{2}^{\mathrm{N}}$ and $I_{0} \ll I_{1}$ and inserting Eq. (38), one gets

$$
\begin{aligned}
& \rho_{0}^{\mathrm{N}}=\rho_{0}-c_{2} \rho_{0} \rho_{2}^{\mathrm{N}} \\
& \rho_{1}^{\mathrm{N}}=\rho_{0}+\left[C-c_{2} \rho_{0}\left\langle l^{2}\right\rangle\right] \rho_{2}^{\mathrm{N}}
\end{aligned}
$$

where $\rho_{0}$ is the density of the isotropic cloud phase at finite, large cutoff, which is still a function of $l_{m}$. The interpretation of these results is that for finite but large $l_{m}$, the cloud and shadow curves differ by terms of or$\operatorname{der} \rho_{2}^{\mathrm{N}}\left(\sim l_{m}^{-1 / 2} \ln l_{m}\right.$ for a log-normal parent), whether expressed in terms of $\rho_{0}$ or $\rho_{1}$. In the limit $l_{m} \rightarrow \infty$, the shadow curve collapses onto the cloud curve, and both tend asymptotically to the limiting form (50). This may appear counterintuitive, suggesting that the nematic phase does not at all "feel" the presence of the orientational order. The answer is of course that only the longer rods in the nematic are orientationally ordered, and for rod lengths comparable to the cutoff $l_{m}$ the nematic and isotropic density distributions do indeed differ. That there is a genuine difference between the isotropic cloud and nematic shadow phases becomes evident if we look at higher order moment densities $\int d l \rho(l) l^{n}$ of the density distributions. In the parent, and therefore the isotropic cloud phase, all these moments will generically be finite (one exception being parent distributions with power-law tails). For the nematic phase, the picture is completely different. For $n=1$ we have the moment $\rho_{1}^{\mathrm{N}}$, and we showed above that the long rod contribution to this converges to zero for $l_{m} \rightarrow \infty$. For larger $n>1$, since the long rod integrals are dominated by values of $l \simeq l_{m}$, the long rod contribution will scale as $l_{m}^{n-1} I_{1} \sim l_{m}^{n-1} \rho_{2}^{\mathrm{N}}$ from Eq. (38) (see App. B). For the log-normal parent, we found that $\rho_{2}^{\mathrm{N}} \sim l_{m}^{-1 / 2} \ln l_{m}$ and so all moments of the nematic shadow phase of order $n>3 / 2$ diverge with $l_{m}$. For parents with less fat tails, $P^{(0)} \sim \exp \left(-l^{\alpha}\right)$, we had instead $\beta \sim l_{m}^{\alpha-1}$ and $\rho_{2}^{\mathrm{N}} \sim \beta^{1 / 2} \sim l_{m}^{(\alpha-1) / 2}$, implying that nematic moments of order $n>(3-\alpha) / 2$ 
diverge. Similarly, moment densities taking account of orientational order, $\int d l \widetilde{d \theta} \rho^{\mathrm{N}}(l, \theta) l^{n} \mathcal{P}_{2}$ diverge when the exponent $n$ is large enough. As we can see then, the similarities between the isotropic cloud phase and the nematic shadow phase are rather more superficial than suggested by the fact that their low-order "physical" moment densities $\rho_{0}$ and $\rho_{1}$ agree, and that $\rho_{2}^{\mathrm{N}} \rightarrow 0$ for $l_{m} \rightarrow \infty$; with respect to any quantity that attaches sufficient weight to the behaviour of the long rods, they are extremely different.

To complete our analysis of the scaling of the moment densities, we need to know in Eqs. $(61,62)$ how the cloud point density $\rho_{0}$ depends on $l_{m}$. By Taylor expanding the relation between $\gamma$ and $\rho_{0}$, Eq. (41), one easily sees that the deviation of $\rho_{0}$ from its limiting value $1 /\left(4\left\langle l^{2}\right\rangle\right)$ is, to lowest order, proportional to $\gamma$. To determine how $\gamma$ converges to zero, compare $\beta=\kappa\left(\rho_{2}^{\mathrm{N}}\right)^{2}$ from Eq. (51) with $\beta=\gamma \rho_{2}^{\mathrm{N}}$ from Eq. (40). This shows that $\gamma$ should be proportional to $\rho_{2}^{\mathrm{N}}$, although we cannot determine the coefficient explicitly: the result $\beta=\gamma \rho_{2}^{\mathrm{N}}$ is only the lowest order in an expansion in $\rho_{2}^{\mathrm{N}}$ and we would need all terms contributing at quadratic order to get the proportionality coefficient in $\gamma \propto \rho_{2}^{\mathrm{N}}$. Nevertheless, $\gamma \propto \rho_{2}^{\mathrm{N}}$ alone tells us that $\rho_{0}$ approaches its limiting value as

$$
\rho_{0}=\frac{1}{4} \frac{1}{\sigma^{2}+1}+\mathcal{O}(\gamma)=\frac{1}{4} \frac{1}{\sigma^{2}+1}+\mathcal{O}\left(\rho_{2}^{\mathrm{N}}\right)
$$

This result, together with Eqs. $(61,62)$ shows that for large $l_{m}$ all the densities $\rho_{0}, \rho_{0}^{\mathrm{N}}$ and $\rho_{1}^{\mathrm{N}}$ differ from their limiting value for $l_{m} \rightarrow \infty$ by terms proportional to $\rho_{2}^{\mathrm{N}}$.

\section{F. Numerical support for the theory}

The analytical results obtained so far are based on the fact that $\beta$ and $\rho_{2}^{\mathrm{N}}$ must converge to zero as the cutoff $l_{m}$ becomes large; we supplemented this with the assumption that $\beta l_{m}$ diverges, from which it then followed that also $\rho_{2}^{\mathrm{N}} l_{m}$ diverges. These statements allowed us to argue that integrals over the rod length $l$ could be split into two distinct parts: one for the short rods, where we could expand in the small parameters $\beta$ and $\rho_{2}^{\mathrm{N}}$, and one for the long rods where the exponential factor $\exp (\beta l)$ is large and dominant and leads to an increase in the nematic density distribution towards the cutoff length. We already saw some graphical support for this in Fig. 5 but provide in this section more detailed numerical support for the theory, focussing on the case of a log-normal parent distribution.

From the theory we obtained that in the limit of infinite cutoff, the cloud and shadow curves collapse onto the curve given by Eq. (50). In other words, the moment densities $\rho_{0}=\rho_{1}$ of the isotropic cloud phase, and those of the nematic phase $\left(\rho_{0}^{\mathrm{N}}\right.$ and $\left.\rho_{1}^{\mathrm{N}}\right)$ should tend to the same limit for $l_{m} \rightarrow \infty$. A plot of these densities against $l_{m}$ (Fig. 10), for fixed polydispersity $\sigma$, shows results compatible with this prediction, with all curves tending towards the theoretically predicted limiting value. A more detailed check is obtained by plotting the densities against $\rho_{2}^{\mathrm{N}}$. Our theory predicts that they should approach their common asymptotic value linearly in $\rho_{2}^{\mathrm{N}}$, and Fig. 11 (a) confirms this. We can even check the known coefficients of this linear dependence by plotting the differences $\rho_{0}-\rho_{0}^{\mathrm{N}}$ and $\rho_{1}^{\mathrm{N}}-\rho_{0}$ against $\rho_{2}^{\mathrm{N}}$. Eqs. $(61,62)$ predict that $\rho_{0}-\rho_{0}^{\mathrm{N}}=c_{2} \rho_{0} \rho_{2}^{\mathrm{N}}$ and $\rho_{1}^{\mathrm{N}}-\rho_{0}=\left[C-c_{2} \rho_{0}\left\langle l^{2}\right\rangle\right] \rho_{2}^{\mathrm{N}}$. In this representation, the $l_{m} \rightarrow \infty$ limits of the coefficients of $\rho_{2}^{\mathrm{N}}$ on the right-hand sides are known, and the unknown proportionality factor in the approach of $\rho_{0}$ to its limiting value (see Eq. (63)) has been eliminated. Figure 11 (b) displays the theoretically predicted linear relations and shows good convergence of the numerical results to these asymptotes. From

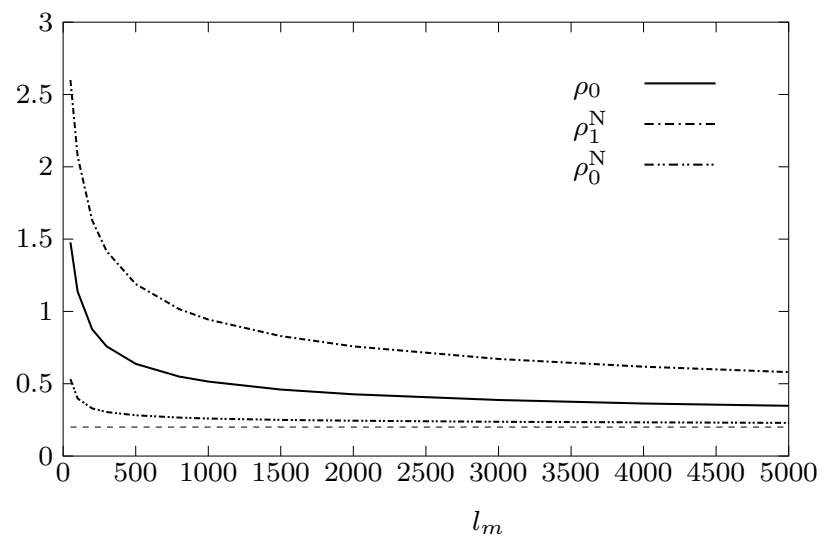

FIG. 10. Plot of the cloud point density $\rho_{0}$ and the nematic shadow density $\rho_{0}^{\mathrm{N}}$ and rescaled volume fraction $\rho_{1}^{\mathrm{N}}$ against the cutoff $l_{m}$, for fixed polydispersity $\sigma \approx 0.532(w=0.5)$. The horizontal line shows the common limiting value for $l_{m} \rightarrow \infty$ which our theory predicts, $1 /\left[4\left(\sigma^{2}+1\right)\right] \approx 0.195$.

the theory we also obtained the scaling of $\beta$ with $l_{m}$, giving $\beta=\left(\ln ^{2} l_{m}\right) /\left(2 l_{m} w^{2}\right)$ to leading order. In Fig. 12 we check this by comparing plots of $\beta$ and $\left(\ln ^{2} l_{m}\right) /\left(2 l_{m} w^{2}\right)$, both against $l_{m}$. For a more detailed comparison, we can estimate from our theory the first correction to the leading behaviour of $\beta$. From Eq. (57) we see for a log-normal parent distribution that this correction term, which arises both from the factor $l_{m}^{2}$ in the denominator and the subleading term in $\ln P^{(0)}\left(l_{m}\right)$, should scale as $\sim l_{m}^{-1} \ln l_{m}$. (Again, because we have not systematically kept track of subleading terms, we do not attempt to determine the coefficient of this correction term.) This correction is smaller by $\mathcal{O}\left(1 / \ln l_{m}\right)$ than the leading term, so that we should have

$$
\frac{\beta l_{m}}{\ln ^{2} l_{m}}=\frac{1}{2 w^{2}}+\mathcal{O}\left(\frac{1}{\ln l_{m}}\right)
$$

and a plot of $\beta l_{m} / \ln ^{2} l_{m}$ against $1 / \ln l_{m}$ should show a linear approach to the limiting value $1 /\left(2 w^{2}\right)$. The results shown in the inset of Fig. 12 are compatible with 


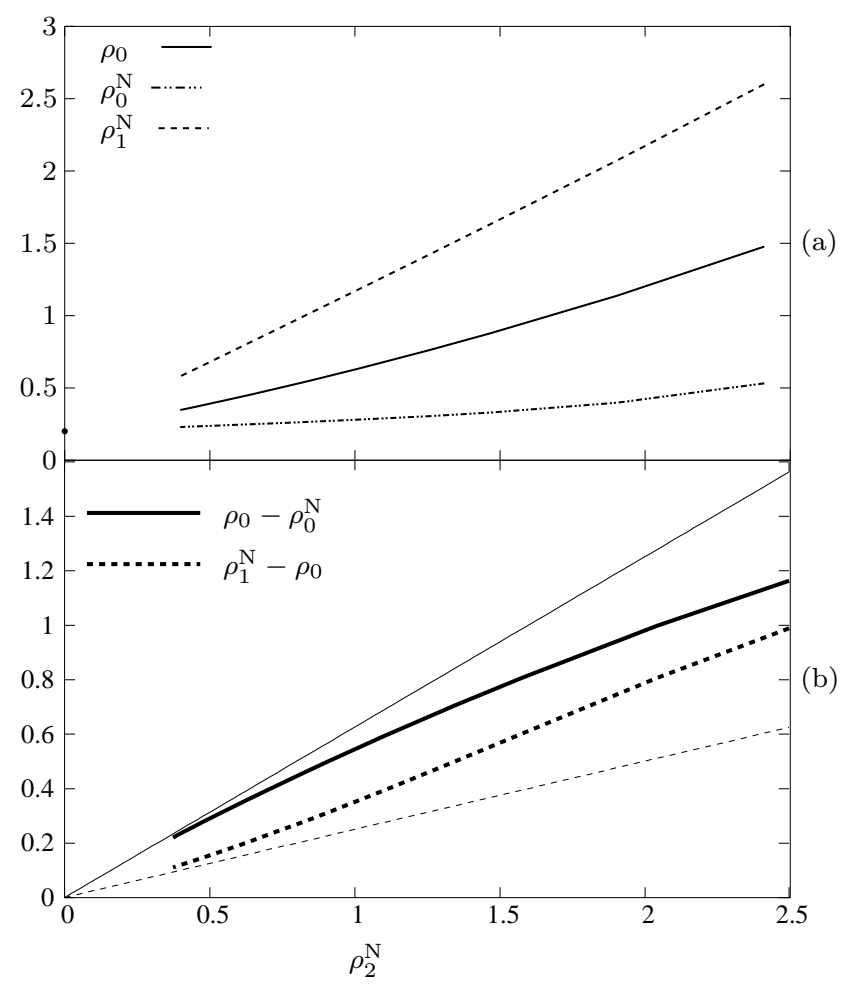

FIG. 11. (a) Same as Fig. 10, but with $\rho_{2}^{\mathrm{N}}$ (which itself is a function of the cutoff $l_{m}$ ) shown on the $x$-axis instead of $l_{m}$ itself. The results are compatible with the theoretically predicted linear approach, for $\rho_{2}^{\mathrm{N}} \rightarrow 0$, to the common limiting value of $1 / 4\left(\sigma^{2}+1\right) \approx 0.195(\sigma \approx 0.532)$ that is marked by the dot. (b) Differences $\rho_{0}-\rho_{0}^{\mathrm{N}}$ (bold solid) and $\rho_{1}^{\mathrm{N}}-\rho_{0}$ (bold dashed) versus $\rho_{2}^{\mathrm{N}}$. For large $l_{m}\left(\right.$ small $\left.\rho_{2}^{\mathrm{N}}\right)$, these converge to the linear relations predicted by Eqs. (61) and (62) and shown by the thin lines.

this. The plot still shows some curvature, however, indicating that even our largest values of $l_{m}=5000$ are not yet large enough for higher-order corrections to be negligible. Accordingly, a linear regression gives an intercept of $\simeq 1.81$, rather below the theoretical value $1 / 2 w^{2}=2$ for the chosen $w=0.5$, while a cubic regression gives a much closer result of $\simeq 1.97$. It is worth stressing that the above results support the assumption $\beta l_{m} \rightarrow \infty$ for $l_{m} \rightarrow \infty$ which we made in our theory: the good fit to the theoretical asymptotics implies that $\beta l_{m} \sim \ln ^{2} l_{m}$ for large $l_{m}$, which indeed diverges. Even for smaller $l_{m}$ the trend for $\beta l_{m}$ to grow with $l_{m}$ is noticeable; e.g. for the scenario shown in Fig. 12, $\beta l_{m}$ increases from $\approx 42$ at $l_{m}=50$ to $\approx 431$ at $l_{m}=5000$.

Finally, our theory also predicts how the parameter $\rho_{2}^{\mathrm{N}}$, giving the overall degree of orientational ordering in the nematic shadow phase, scales with $l_{m}$. The result is that $\rho_{2}^{\mathrm{N}}$ should be proportional to $\beta^{1 / 2}$ for large $l_{m}$, so that a plot of these two quantities against each other as $l_{m}$ is varied should give a straight line through the origin, with slope $\kappa^{-1 / 2}$ from Eq. (51). Fig. 13 is consistent with this,

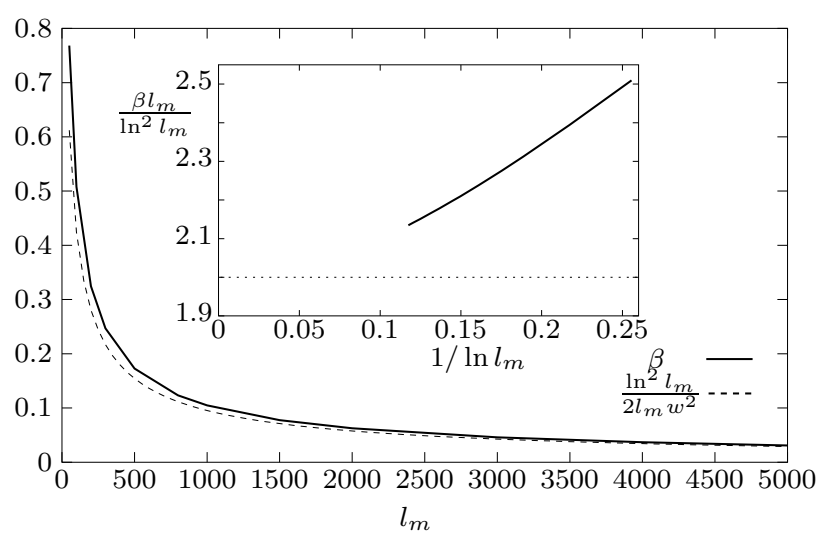

FIG. 12. Plot of $\beta$ (solid) and the theoretically predicted asymptotic scaling $\beta=\left(\ln ^{2} l_{m}\right) /\left(2 w^{2}\right)$ (dashed) against $l_{m}$ at $w=0.5$. The inset provides a more stringent test of the theory by plotting $\beta l_{m} / \ln ^{2} l_{m}$ versus $1 / \ln l_{m}$. Theory predicts that this should approach $1 / 2 w^{2}=2$ (dotted line) linearly; the results plotted are broadly compatible with this but still show some curvature, indicating that our values of $l_{m}$ are still too small to have reached the asymptotic regime where higher-order corrections can be neglected.

but shows the expected deviations in the pre-asymptotic regime where $\rho_{2}^{\mathrm{N}}$ and $\beta$ are not yet small.

\section{CONCLUSION}

We have analysed the effects of length polydispersity on the phase behaviour of hard rods, within the $\mathcal{P}_{2}$ Onsager model which is a simplified version of the full Onsager theory. We focussed in particular on rod length distributions with fat tails that decay less than exponentially, using log-normal distributions as our primary example. Our work was originally motivated by the question of whether, even for such unimodal length distributions, the presence of relatively many long rods could cause I-N-N phase separation to occur. We do indeed find an I-N-N coexistence region in the phase diagram for a log-normal length distribution with a finite cutoff. Unusually, this region is located inside the I-N coexistence region, i.e. not connected to an $\mathrm{N}-\mathrm{N}$ region, and very narrow in density, separating two different regimes of isotropic-nematic coexistence. At densities below the three-phase region, the nematic phase is significantly enriched in long rods compared to the isotropic: its density distribution exhibits a second peak for large rod lengths, in addition to the one at lengths of order unity (the average rod length in the system). Because the overall number of long rods in the system is small, however, the nematic phase only occupies a very small part of the system volume in this regime. As the density increases towards the three-phase region, the second peak in the nematic length distribution moves towards the main peak; beyond 


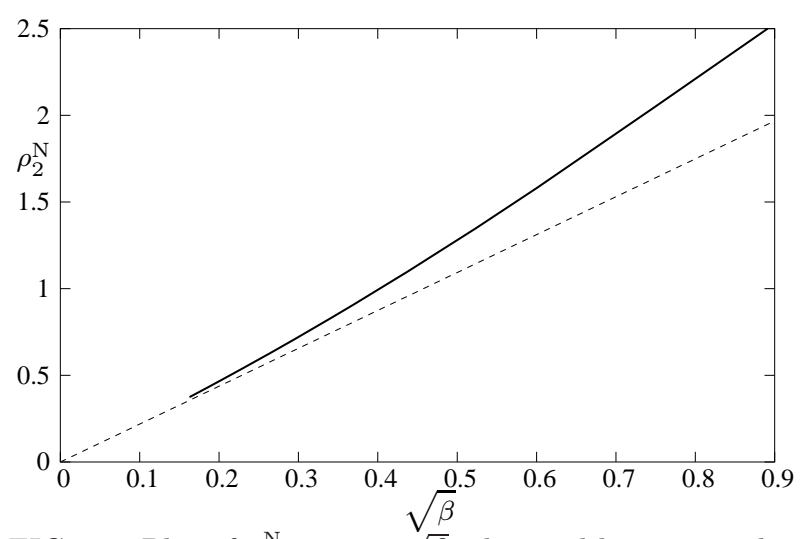

FIG. 13. Plot of $\rho_{2}^{\mathrm{N}}$ against $\sqrt{\beta}$, obtained by varying $l_{m}$ at fixed $w=0.5$ (solid), together with the asymptotic scaling $\rho_{2}^{\mathrm{N}}=(\beta / \kappa)^{1 / 2}$ predicted from Eq. (51) (dashed). As predicted by theory, both $\beta$ and $\rho_{2}^{\mathrm{N}}$ tend towards zero as $l_{m}$ increases. In the same limit they also approach the predicted linear asymptote, while for smaller $l_{m}$ and therefore larger $\rho_{2}^{\mathrm{N}}$ and $\beta$ deviations from linearity occur.

the three-phase region, the coexisting nematic only exhibits a single peak, and the phase behaviour resembles that found for systems without fat-tailed length distributions. Moving towards larger polydispersities in the phase diagram, the three-phase region eventually closes off and is replaced by a crossover, within the I-N coexistence region, between the two types of nematic phase just described. Coming from small polydispersity $\sigma$, on the other hand, the three-phase region begins at a threshold value of $\sigma$ where the isotropic cloud curve shows a kink and the properties of the coexisting nematic shadow phase change discontinuously.

In the theoretical part of the paper, we gave an analytical treatment of the onset of nematic order, i.e. of the isotropic cloud point, in the limit of large length cutoffs $l_{m}$. The key quantities for the theory were the parameter $\beta$, which determines the exponential enrichment of the nematic phase in the longer rods, and the average ordering $\rho_{2}^{\mathrm{N}}$ of the nematic phase. We were able to show that both of these quantities must converge to zero for $l_{m} \rightarrow \infty$, and based on our numerical results supplemented this with the assumption that $\beta l_{m} \rightarrow \infty$. On this basis we could construct a self-consistent theory for the limit of large $l_{m}$, by treating separately the behaviour of short rods (with lengths of order one) and long rods of order the cutoff length. Surprisingly, the theory predicts that the density (and rescaled rod volume fraction) $\rho_{0}$ of the isotropic phase, and the density $\rho_{0}^{\mathrm{N}}$ and rescaled rod volume fraction $\rho_{1}^{\mathrm{N}}$ of the nematic shadow phase, all converge to the same limiting curve when plotted against polydispersity $\sigma$. This curve can be calculated explicitly and depends on no other details of the rod length distribution except for the presence of the fat tail. The theory in fact makes a stronger statement about the similarity of the isotropic cloud and nematic shadow phases: for any rod length of order unity, the density distributions in the two phases become equal for $l_{m} \rightarrow \infty$, and the degree of orientational order of such rods in the nematic shadow tends to zero. This does not, however, imply that the isotropic and nematic phases coincide completely, or that the nematic lacks orientational order: when we consider the longest rods in the system, of the order of the cutoff length, we find that the nematic shadow contains a much larger number of them, which also exhibit strong nematic order. Our theory also allowed us ascertain the rate of convergence of all quantities to their limit values, to leading order as $l_{m} \rightarrow \infty$, and the predictions were in good agreement with numerical results.

An important question that our current study cannot answer directly is how the above results for the $\mathcal{P}_{2}$ Onsager model will carry over to the full Onsager theory. The comparison of our earlier results for bidisperse distributions $^{30}$ were encouraging in this respect, showing qualitative agreement except for the presence of an N$\mathrm{N}$ critical point in the $\mathcal{P}_{2}$ Onsager model which in the Onsager theory is replaced by an unbounded N-N coexistence region opening towards large densities. A direct attack on the full Onsager problem, with the required selfconsistent determination of all orientation distributions, is of course a formidable numerical problem. Nevertheless, we have recently obtained preliminary results which suggest that the isotropic cloud curve for Onsager theory with a log-normal length distribution indeed features a kink, as it does for the $\mathcal{P}_{2}$ Onsager model studied above; this shows indirectly that the full Onsager theory must also exhibit an I-N-N coexistence region as found above. The overall topology of the phase diagram remains an open question, however; in particular, one would like to know whether the I-N-N coexistence is again confined to a narrow interval or whether it extends over a broader range and is bordered by an N-N phase separation at higher densities.

Acknowledgements: PS is grateful for financial support through EPSRC grant GR/R52121/01.

\section{APPENDIX A: THE CASE $\beta \rightarrow 0, \rho_{2}^{N}$ FINITE}

In our theory for the isotropic cloud point we showed that in the limit of large cutoff $l_{m} \rightarrow \infty$, the parameter $\beta$ tends to zero while $\beta l_{m} \rightarrow \infty$. The moment density $\rho_{2}^{\mathrm{N}}$, on the other hand, which describes the orientational ordering of the nematic shadow phase, could in principle either also tend to zero, or converge to a nonzero constant in the limit. In this appendix we show that the second case can be excluded because it does not lead to a consistent solution of the cloud point equations.

The assumption that $\beta \rightarrow 0$ again allows us to break all integrals over $l$ into two intervals, $l=0 \ldots \tilde{l}$ and $l=$ $\tilde{l} \ldots l_{m}$ with $1 \ll \tilde{l} \ll l_{m}$. All statements made in the main text regarding the long rod contributions $I_{0}, I_{1}$ and $I_{2}$ to the nematic shadow phase moments $\rho_{0}^{\mathrm{N}}, \rho_{1}^{\mathrm{N}}$ and $\rho_{2}^{\mathrm{N}}$ 
also remain valid, seeing as the only property of $\rho_{2}^{\mathrm{N}}$ that entered was that $\rho_{2}^{\mathrm{N}} l_{m}$ diverges as $l_{m} \rightarrow \infty$; if $\rho_{2}^{\mathrm{N}}$ itself has a nonzero limit value then this is certainly the case. We know therefore that $I_{0}$ is smaller by a factor $1 / l_{m}$ than $I_{1}$ and can be neglected, and that $I_{2}=I_{1}$ to leading order since as a consequence of $\rho_{2}^{\mathrm{N}} l_{m} \rightarrow \infty$ the longest rods are strongly ordered.

The treatment of the short rod parts is actually easier than in the case of $\rho_{2}^{N} \rightarrow 0$ : a finite limit value of $\rho_{2}^{N}$ ensures that these integrals have nonvanishing differences from the corresponding values in the isotropic phase, even for $l_{m} \rightarrow \infty$. Any corrections from the small nonzero value of $\beta$ are negligible by comparison, so that we can set $\beta=0$ directly and write Eqs. $(22,23,24)$ as

$$
\begin{aligned}
& \rho_{0}^{\mathrm{N}}=\rho_{0} J_{0} \\
& \rho_{1}^{\mathrm{N}}=\rho_{0}\left(J_{1}+I_{1}^{\prime}\right) \\
& \rho_{2}^{\mathrm{N}}=\rho_{0}\left(J_{2}+I_{1}^{\prime}\right)
\end{aligned}
$$

where $I_{1}^{\prime}=I_{1} / \rho_{0}$ and

$$
\begin{aligned}
& J_{0}=\int d l \widetilde{d \theta} P^{(0)}(l) e^{f(\theta) l} \\
& J_{1}=\int d l \widetilde{d \theta} l P^{(0)}(l) e^{f(\theta) l} \\
& J_{2}=\int d l \widetilde{d \theta} l P^{(0)}(l) \mathcal{P}_{2} e^{f(\theta) l}
\end{aligned}
$$

(In principle, the short rod integrals run over $l=0 \ldots \tilde{l}$, but because the integrals as written are convergent, one can take the upper limit to $\infty$ without error at the level of our approximation.) We can determine the value of $I_{1}^{\prime}$ from Eq. (27) for $\beta$, which using $\beta \rightarrow 0$ becomes

$$
0=-c_{1}\left(\rho_{1}^{\mathrm{N}}-\rho_{0}\right)+c_{2} \rho_{2}^{\mathrm{N}}
$$

and solving for $I_{1}^{\prime}$ one finds

$$
I_{1}^{\prime}=\frac{c_{1}-c_{1} J_{1}+c_{2} J_{2}}{c_{1}-c_{2}}
$$

Inserting this into the pressure equality (26), which with a common factor of $\rho_{0}$ removed reads

$$
1+\frac{c_{1}}{2} \rho_{0}=J_{0}+\frac{c_{1}}{2} \rho_{0}\left(J_{1}+I_{1}^{\prime}\right)^{2}-\frac{c_{2}}{2} \rho_{0}\left(J_{2}+I_{1}^{\prime}\right)^{2}
$$

we can solve explicitly to obtain the cloud point density $\rho_{0}$ as

$$
\rho_{0}=\frac{c_{1}-c_{2}}{c_{1} c_{2}} \frac{2\left(1-J_{0}\right)}{1-\left(J_{1}-J_{2}\right)^{2}}
$$

where the r.h.s. is now a function only of $\rho_{2}^{\mathrm{N}}$, via the short rod integrals $J_{0}, J_{1}$ and $J_{2}$. Finally, substituting the results (A2) for $I_{1}^{\prime}$ and (A3) for $\rho_{0}$ into Eq. (A1) for $\rho_{2}^{\mathrm{N}}$, we obtain after a little algebra the self-consistency equation

$$
\rho_{2}^{\mathrm{N}}=F\left(\rho_{2}^{\mathrm{N}}\right)
$$

where

$$
F\left(\rho_{2}^{\mathrm{N}}\right)=\frac{2\left(1-J_{0}\right)}{c_{2}\left(J_{1}-J_{2}+1\right)}
$$

It can be shown that this function passes through the origin with a slope of one, but then curves downwards and approaches a constant for large $\rho_{2}^{\mathrm{N}}$. It follows that

$$
F\left(\rho_{2}^{\mathrm{N}}\right)<\rho_{2}^{\mathrm{N}}
$$

for $\rho_{2}^{\mathrm{N}}>0$, so that the only solution is $\rho_{2}^{\mathrm{N}}=0$, in contradiction to our assumption that $\rho_{2}^{\mathrm{N}}$ had a nonzero limit value for $l_{m} \rightarrow \infty$. This assumption must therefore be abandoned, and we conclude that $\rho_{2}^{\mathrm{N}}$ must tend to zero for $l_{m} \rightarrow \infty$. From our arguments it is clear that this conclusion holds for any fat-tailed parent distribution.

We mention finally that, even though the above treatment based on the assumption of nonzero $\rho_{2}^{\mathrm{N}}$ is not fully self-consistent, it actually recovers the correct limiting value of the cloud point density. As pointed out above, Eq. (A4) has $\rho_{2}^{\mathrm{N}}=0$ as its only solution. Now for small $\rho_{2}^{\mathrm{N}}$ one can expand $J_{0}=1-c_{2} \rho_{2}^{\mathrm{N}}, J_{1}=1-c_{2} \rho_{2}^{\mathrm{N}}\left\langle l^{2}\right\rangle$ and $J_{2}=\left(c_{2} / 5\right) \rho_{2}^{\mathrm{N}}\left\langle l^{2}\right\rangle$; inserting these results into Eq. (A3) one finds $\rho_{0}=1 /\left(4\left\langle l^{2}\right\rangle\right)$ in the limit $\rho_{2}^{\mathrm{N}} \rightarrow 0$, in agreement with Eq. (50).

\section{APPENDIX B: THE LONG ROD INTEGRALS}

In this appendix we discuss how to evaluate the general long rod integral

$$
I=\rho_{0} \int_{\tilde{l}}^{l_{m}} d l P(l) l^{n} e^{\beta l} \int \widetilde{d \theta} e^{l f(\theta)}
$$

with $P(l) \equiv P^{(0)}(l)$ for brevity. For $n=0$ and 1 this reduces to $I_{0}$ and $I_{1}$, the long rod contributions to the moments $\rho_{0}^{\mathrm{N}}$ and $\rho_{1}^{\mathrm{N}}$ of the nematic shadow phase. As explained before Eq. (52), since the integral will turn out to be dominated by the long rods with $l \approx l_{m}$, we can use the fact that these rods are strongly ordered $\left(\rho_{2}^{\mathrm{N}} l \gg 1\right)$ to perform the angular integral and get

$$
I=\frac{\rho_{0}}{3 c_{2} \rho_{2}^{\mathrm{N}}} \tilde{I}, \quad \tilde{I}=\int_{\tilde{l}}^{l_{m}} d l P(l) l^{n-1} e^{\beta l}
$$

Our task now is to evaluate $\tilde{I}$. If this really is dominated by $l \approx l_{m}$ due to the presence of the exponential factor $\exp (\beta l)$, we should be able to replace the rest of the integrand, $f(l)=P(l) l^{n-1}$ by its value at $l=l_{m}$. To check the quality of this approximation, we include the first term in a Taylor expansion,

$$
f(l)=f\left(l_{m}\right)+\left(l-l_{m}\right) f^{\prime}\left(l_{m}\right)+\ldots
$$

which when inserted into Eq. (B1) gives

$$
\tilde{I}=\left[f\left(l_{m}\right)-\frac{f^{\prime}\left(l_{m}\right)}{\beta}\right] \frac{e^{\beta l_{m}}}{\beta}
$$


If the leading term is a good approximation, the correction term should be small, i.e. $-f^{\prime}\left(l_{m}\right) /\left[\beta f\left(l_{m}\right)\right] \rightarrow 0$ for $l_{m} \rightarrow \infty$. Whether this is true depends on the $l_{m^{-}}$ dependence of $\beta$. From Eq. (54), this is given implicitly by $\tilde{I} / \beta=A$, with $A$ a constant and $\tilde{I}$ evaluated at $n=1$, i.e. for $f(l)=P(l)$. Keeping the leading term of $\tilde{I}$ only, we get

$$
\frac{e^{\beta l_{m}}}{\left(\beta l_{m}\right)^{2}}=\frac{A}{P\left(l_{m}\right) l_{m}^{2}}
$$

Using the inverse of the function $G(x)=e^{x} / x^{2}$ on the 1.h.s. this can be formally solved to give

$$
\beta=\frac{1}{l_{m}} G^{-1}\left(\frac{A}{P\left(l_{m}\right) l_{m}^{2}}\right)
$$

Now from the definition of $G(x)$ we have

$$
G(x)=y \quad \Rightarrow \quad x=\ln y+2 \ln x
$$

and therefore by recursion

$$
x=\ln y+2 \ln (\ln y+2 \ln (\ln y+2 \ln \ldots))
$$

Asymptotically the first term is dominant, so that $x=$ $G^{-1}(y)=\ln y$ and the asymptotic behaviour of $\beta$ becomes

$$
\beta=\frac{1}{l_{m}} \ln \left(\frac{A}{P\left(l_{m}\right) l_{m}^{2}}\right)
$$

The condition $-f^{\prime}\left(l_{m}\right) /\left[\beta f\left(l_{m}\right)\right] \rightarrow 0$ for our leading order-approximation for $\tilde{I}$ to be correct is thus

$$
-\frac{l_{m} f^{\prime}\left(l_{m}\right)}{f\left(l_{m}\right)\left\{\ln A-\ln \left[P\left(l_{m}\right) l_{m}^{2}\right]\right\}} \rightarrow 0
$$

If we now restrict our attention to parent distributions with finite mean, i.e. $\int d l P(l) l<\infty$, then $P\left(l_{m}\right) l_{m}^{2} \rightarrow 0$ for $l_{m} \rightarrow \infty$ and the constant term $\ln A$ becomes negligible. We therefore need

$$
\frac{l_{m}(\ln f)^{\prime}\left(l_{m}\right)}{\ln \left[P\left(l_{m}\right) l_{m}^{2}\right]}=\frac{l_{m}\left[(\ln P)^{\prime}\left(l_{m}\right)+(n-1) / l_{m}\right]}{\ln \left[P\left(l_{m}\right) l_{m}^{2}\right]} \rightarrow 0
$$

For a log-normal parent distribution, $\ln P(l)=$ $-\left(\ln ^{2} l\right) /\left(2 w^{2}\right)$ for large $l$ and so $(\ln P)^{\prime}(l)=$ $-(\ln l) /\left(w^{2} l\right)$, and the condition becomes

$$
\frac{l_{m}\left[\left(\ln l_{m}\right) / l_{m}+\mathcal{O}\left(l_{m}^{-1}\right)\right]}{\left(\ln ^{2} l_{m}\right)^{2} / 2+\mathcal{O}\left(\ln l_{m}\right)} \simeq \frac{2}{\ln l_{m}} \rightarrow 0
$$

which is obviously fulfilled for $l_{m} \rightarrow \infty$. For power-law parents, $\ln P(l) \sim \ln l$, one also finds that the ratio (B7) tends to zero logarithmically. In both these cases, one is therefore justified in only keeping the leading term in our Taylor expansion around $l=l_{m}$, giving

$$
\tilde{I}=\frac{f\left(l_{m}\right) e^{\beta l_{m}}}{\beta}=\frac{P\left(l_{m}\right) l_{m}^{n-1} e^{\beta l_{m}}}{\beta}
$$

This proves in particular the statement made in the main text that the effect of the weight function $l^{n}$ only comes in through the factor $l_{m}^{n}$. It is also worth stressing that Eq. (B5) implies that $\beta l_{m} \simeq-\ln \left[P\left(l_{m}\right) l_{m}^{2}\right] \rightarrow \infty$, consistent with our assumption of such a divergence. This result also justifies a posteriori the intuition that the integrals for $I$ and $\tilde{I}$ are dominated by the longest rods: the exponential integral giving the leading order approximation has significant contributions only where $l_{m}-l$ is of order $1 / \beta$, so that the fractional deviation of the relevant $l$-values from $l_{m},\left(l_{m}-l\right) / l_{m} \sim 1 /\left(\beta l_{m}\right)$ tends to zero for $l_{m} \rightarrow \infty$.

In the more general case of a less than exponentially decaying parent, say $P(l)=e^{-h(l)}$ and $h(l) \sim l^{\alpha}$ with $0<$ $\alpha<1$, we have $(\ln P)^{\prime}(l) \sim l^{\alpha-1}$ and the condition (B7) becomes

$$
\frac{l_{m}\left[\alpha l_{m}^{\alpha-1}+\mathcal{O}\left(l_{m}^{-1}\right)\right]}{l_{m}^{\alpha}+\mathcal{O}\left(\ln l_{m}\right)} \simeq \alpha \neq 0
$$

Here one therefore needs to go beyond the leading order approximation for $\tilde{I}$. To do so, one can write $f(l)=$ $\exp [-h(l)+(n-1) \ln l]$ and keep in Eq. (B1) linear terms in the Taylor expansion of the exponent around $l=l_{m}$. This results in

$$
\begin{aligned}
\tilde{I} & =\int_{\tilde{l}}^{l_{m}} d l e^{-h\left(l_{m}\right)+(n-1) \ln l_{m}+\beta l_{m}+\left(l-l_{m}\right)\left[\beta-h^{\prime}\left(l_{m}\right)+(n-1) / l_{m}\right]} \\
& =\frac{l_{m}^{n-1} e^{-h\left(l_{m}\right)+\beta l_{m}}}{\beta-h^{\prime}\left(l_{m}\right)+(n-1) / l_{m}}
\end{aligned}
$$

For $n=1$ this gives Eq. (59) in the main text. (The term $(n-1) / l_{m}$ in the denominator can be neglected even for $n \neq 1$ since, having dealt with the power-law distributions case $h(l) \sim \ln l$ above, we can here assume that $h(l)$ diverges more strongly than $\ln l$.) From this one can again deduce the scaling of $\beta$, using the condition $\tilde{I} / \beta=A$

$$
\frac{e^{-h\left(l_{m}\right)+\beta l_{m}}}{\beta\left[\beta-h^{\prime}\left(l_{m}\right)\right]}=A
$$

To leading order this gives Eq. $(60), \beta=h\left(l_{m}\right) / l_{m}$; this can be shown by writing $\beta=h\left(l_{m}\right) / l_{m}+\delta$, substituting into the above condition and verifying that the assumption that $\delta$ is a small correction is self-consistent. Once the scaling of $\beta$ is established, one can then show that higher-order corrections to the first-order approximation (B8) are indeed vanishingly small as $l_{m} \rightarrow \infty$; we omit the details here.

${ }^{1}$ H Z Zocher. Anorg. Chem., 147:91, 1925. 
${ }^{2}$ F C Bawden, N W Pirie, J D Bernal, and I Fankuchen. Liquid crystalline substances from virus-infected plants. $\mathrm{Na}$ ture, 138:1051, 1936.

${ }^{3} \mathrm{P}$ A Buining, C Pathmamanoharan, A P Philipse, and H N W Lekkerkerker. Preparation of (non-)aqueous dispersions of colloidal boehmite needles. Chem. Eng. Sci., 48(2):411-417, 1993.

${ }^{4} \mathrm{P}$ A Buining, Y S J Veldhuizen, C Pathmamanoharan, and H N W Lekkerkerker. Preparation of a nonaqueous dispersion of sterically stabilized boehmite rods. Colloid Surface, 64(1):47-55, 1992.

${ }^{5} \mathrm{M}$ P B van Bruggen, F M van der Kooij, and $\mathrm{H} \mathrm{N}$ W Lekkerkerker. Liquid crystal phase transitions in dispersions of rod-like colloidal particles. J. Phys.-Condens. Matter, 8(47):9451-9456, 1996.

${ }^{6} \mathrm{~W}$ Maier and A Saupe. Z. Naturforsch. A, 13a:564, 1958.

${ }^{7} \mathrm{~L}$ Onsager. The effect of shape on the interaction of colloidal particles. Ann. N.Y. Acad. Sci, 51:627, 1949.

${ }^{8} \mathrm{H}$ N W Lekkerkerker, P Coulon, $\mathrm{R}$ van der Haegen, and R Deblieck. On the isotropic-liquid crystal phaseseparation in a solution of rodlike particles of different lengths. J. Chem. Phys., 80(7):3427-3433, 1984.

${ }^{9}$ Richard F Jr Kayser and Harold Raveché. Bifurcation in onsager's model of the isotropic-nematic transition. Phys. Rev. A, 17:2067-2072, 1978.

${ }^{10}$ A R Khokhlov and A N Semenov. Liquid-crystalline ordering in the solution of long persistent chains. Physica A, 108A:546-555, 1981.

${ }^{11}$ A R Khokhlov and A N Semenov. Liquid-crystalline ordering in the solution of partially flexible macromolecules. Physica A, 112A:605-614, 1981.

${ }^{12} \mathrm{P}$ A Buining and $\mathrm{H} \mathrm{N} \mathrm{W}$ Lekkerkerker. Isotropic-nematic phase-separation of a dispersion of organophilic boehmite rods. J. Phys. Chem., 97(44):11510-11516, 1993.

${ }^{13} \mathrm{P}$ Sollich. Predicting phase equilibria in polydisperse systems. J. Phys.-Condes. Matter, 14(3):R79-R117, 2002.

${ }^{14}$ T Odijk and H N W Lekkerkerker. Theory of the isotropic liquid-crystal phase-separation for a solution of bidisperse rodlike macromolecules. J. Phys. Chem., 89(10):2090-2096, 1985.

${ }^{15} \mathrm{R}$ van Roij and B Mulder. Absence of high-density consolute point in nematic hard rod mixtures (vol 105, pg 11237, 1996). J. Chem. Phys., 105:11237, 1996.

${ }^{16} \mathrm{G}$ J Vroege and H N W Lekkerkerker. Theory of the isotropic nematic nematic phase-separation for a solution of bidisperse rodlike particles. J. Phys. Chem., 97(14):36013605, 1993.

17 Zheng Yu Chen. Effect of polydispersity on the isotropicnematic phase transition of rigid rods. Phys. Rev. E, 50:2849-2855, 1994.

${ }^{18}$ R M L Evans. Fractionation of polydisperse systems: multiphase coexistence. Phys. Rev. E, 59(3):3192-3195, 1999.

${ }^{19}$ T J Sluckin. Polydispersity in liquid-crystal systems. Liq. Cryst., 6(1):111-131, 1989.

${ }^{20}$ N Clarke, J A Cuesta, R Sear, P Sollich, and A Speranza. Phase equilibria in the polydisperse zwanzig model of hard rods. J. Chem. Phys., 113(14):5817-5829, 2000.

${ }^{21} \mathrm{R}$ Zwanzig. First-order phase transition in a gas of long thin rods. J. Chem. Phys., 39:1714, 1963.

${ }^{22}$ P J Flory. Statistical thermodynamics of semi-flexible chain molecules. Proc. R. Soc. London A, 234:60, 1956.

${ }^{23} \mathrm{P}$ J Flory and A Abe. Statistical thermodynamics of mixtures of rodlike particles. 1. Theory for polydisperse systems. Macromolecules, 11:1119, 1978.

${ }^{24}$ A Abe and P J Flory. Statistical Thermodynamics of Mixtures of Rodlike Particles. 2. Ternary Systems. Macromolecules, 11:1122, 1978.

${ }^{25}$ P J Flory and R S Frost. Statistical Thermodynamics of Mixtures of Rodlike Particles. 3. The Most Probable Distribution. Macromolecules, 11:1126, 1978.

${ }^{26}$ R S Frost and P J Flory. Statistical Thermodynamics of Mixtures of Rodlike Particles. 4. The Poisson Distribution. Macromolecules, 11:1134, 1978.

${ }^{27} \mathrm{~J} \mathrm{~K}$ Moscicki and G Williams. The effect of a Gaussian distribution of chain-lengths on the phase behaviour of a model system of rod-like macromolecules in solution. Polymer, 23:558, 1982.

${ }^{28}$ T M Birshtein, B I Kolegov, and V A Pryamistsyn. Theory of athermal lyotropic liquid crystal systems. Polymer Sci. U.S.S.R., 30(2):316-324, 1986.

${ }^{29} \mathrm{~T}$ M Birshtein, B I Kolegov, and V A Pryamitsin. On theory of athermal lyotropic liquid-crystalline systems. Vysokomol Soedin Ser A, 30(2):348-354, 1988.

${ }^{30}$ A Speranza and P Sollich. Simplified onsager theory for isotropic-nematic phase equilibria of polydisperse hard rods. J. Chem. Phys., to appear.

${ }^{31} \mathrm{~K}$ Šolc. Cloud-point curves of polymers solutions. Macromolecules, 3(5):665-673, 1970.

${ }^{32} \mathrm{~K}$ Šolc. Cloud-point curves of polymers with logarithmicnormal distribution of molecular weight. Macromolecules, 8(6):819-827, 1975.

${ }^{33} \mathrm{P}$ Sollich and M E Cates. Projected free energies for polydisperse phase equilibria. Phys. Rev. Lett., 80(7):13651368, 1998.

${ }^{34}$ P Sollich, P B Warren, and M E Cates. Moment free energies for polydisperse systems. Adv. Chem. Phys., 116:265336, 2001.

${ }^{35} \mathrm{P}$ B Warren. Combinatorial entropy and the statistical mechanics of polydispersity. Phys. Rev. Lett., 80(7):13691372, 1998.

${ }^{36}$ In the following we will use the same convention as we used in the numerical solution of the phase coexistence equations, i.e., we will assume that the functional form of $P^{(0)}(l)$ is fixed as $l_{m}$ is varied. In this way the parent will be strictly normalized only in the limit for $l_{m} \rightarrow \infty$, and the average length will be strictly equal to 1 in the same limit. However, as we mentioned in Sec. III, the corrections to the strict normalization are small for sufficiently large cutoff. In the following, as we are interested in the large cutoff limit, we will therefore always assume $\rho_{1}=\rho_{0}$ for the parent distribution. 